\title{
Culture and identification of Borrelia spirochetes in human
}

\section{vaginal and seminal secretions [version 1; peer review: 1 not}

\section{approved]}

\author{
Marianne J. Middelveen ${ }^{1}$, Jennie Burke ${ }^{2}$, Eva Sapi ${ }^{3}$, Cheryl Bandoski ${ }^{3}$, \\ Katherine R. Filush ${ }^{3}$, Yean Wang ${ }^{2}$, Agustin Franco ${ }^{2}$, Arun Timmaraju ${ }^{3}$, \\ Hilary A. Schlinger ${ }^{1}$, Peter J. Mayne ${ }^{1}$, Raphael B. Stricker ${ }^{1}$ \\ ${ }^{1}$ International Lyme and Associated Diseases Society, Bethesda, MD, 20827-1461, USA \\ ${ }^{2}$ Australian Biologics, Sydney, NSW 2000, Australia \\ ${ }^{3}$ Department of Biology and Environmental Science, University of New Haven, West Haven, CT, 06516, USA
}

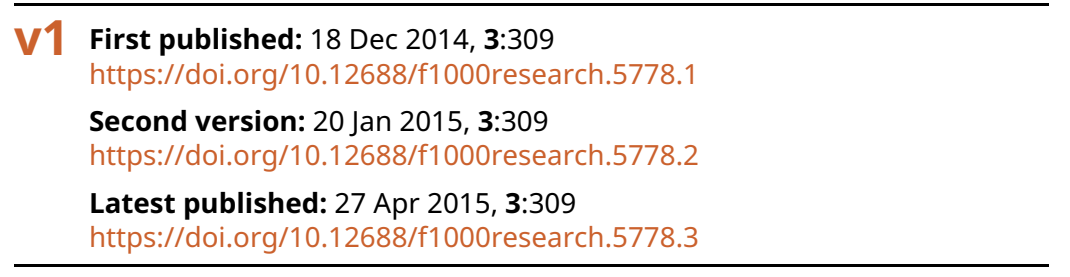

\section{Abstract}

Background: Recent reports indicate that more than 300,000 cases of Lyme disease are diagnosed yearly in the USA. Preliminary clinical, epidemiological and immunological studies suggest that infection with the Lyme disease spirochete Borrelia burgdorferi $(\mathrm{Bb})$ could be transferred from person to person via intimate human contact without a tick vector. Detecting viable Borrelia spirochetes in vaginal and seminal secretions would provide evidence to support this hypothesis.

Methods: Patients with and without a history of Lyme disease were selected for the study after informed consent was obtained. Serological testing for $\mathrm{Bb}$ was performed on all subjects. Semen or vaginal secretions were inoculated into BSK-H medium and cultured for four weeks. Examination of genital cultures and culture concentrates for the presence of spirochetes was performed using light and darkfield microscopy, and spirochete concentrates were subjected to Dieterle silver staining, anti-Bb immunohistochemical staining, molecular hybridization and PCR analysis for further characterization. Immunohistochemical and molecular testing was performed in three independent laboratories. Positive and negative controls were included in all experiments.

Results: Control subjects who were asymptomatic and seronegative for $\mathrm{Bb}$ had no detectable spirochetes in genital secretions by PCR analysis. In contrast, spirochetes were observed in cultures of genital secretions from 11 of 13 subjects diagnosed with Lyme disease, and motile spirochetes were detected in genital culture concentrates from 12 of 13 Lyme disease patients using light and darkfield microscopy.

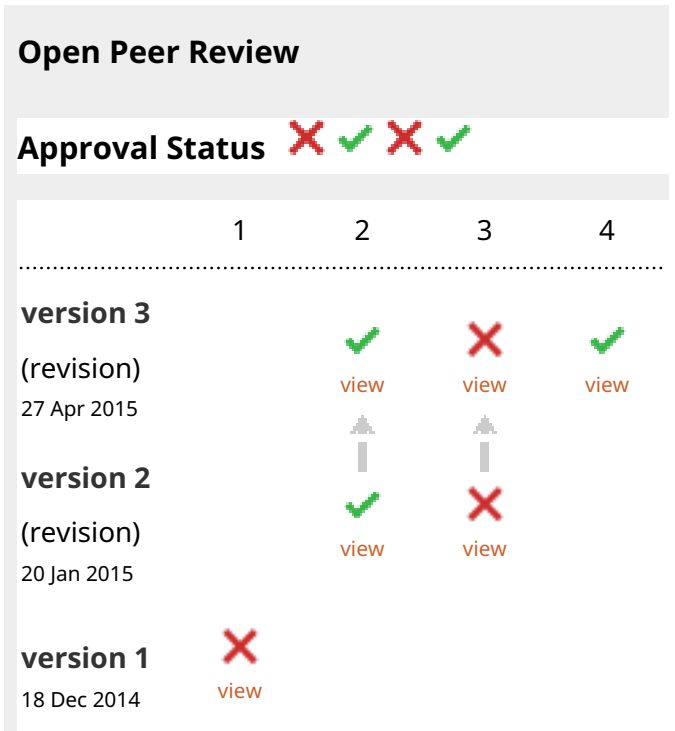

1. Sam T. Donta, Falmouth Hospital, Falmouth, USA

2. Robert A Smith, University of Glasgow, Glasgow, UK

3. Monica E. Embers, Tulane University Health Sciences, Covington, USA

4. Nataliia Rudenko ID, Biology Centre Czech Academy of Sciences, České Budějovice, Czech Republic

Maryna Golovchenko, Biology Centre Czech 
Morphological features of spirochetes were confirmed by Dieterle silver staining and immunohistochemical staining of culture concentrates. Molecular hybridization and PCR testing confirmed that the spirochetes isolated from semen and vaginal secretions were strains of Borrelia, and all cultures were negative for treponemal spirochetes. PCR sequencing of cultured spirochetes from three couples having unprotected sex indicated that two couples had identical strains of $\mathrm{Bb}$ sensu stricto in their semen and vaginal secretions, while the third couple had identical strains of $B$. hermsii detected in their genital secretions.

Conclusions: The culture of viable Borrelia spirochetes in genital secretions suggests that Lyme disease could be transmitted by intimate contact from person to person.

Keywords

Lyme borreliosis, chronic Lyme disease, Borrelia burgdorferi, spirochetes, sexual transmission.
Academy of Sciences, České Budějovice,

Czech Republic

Any reports and responses or comments on the article can be found at the end of the article.

Corresponding author: Raphael B. Stricker (rstricker@usmamed.com)

Competing interests: The authors have no competing interests to declare. Preliminary results of the study were presented at the Western Regional Meeting of the American Federation for Medical Research, Carmel, CA, on January 25, 2014, and published in abstract form ( Invest Med 2014;62:280-1).

Grant information: Supported in part by a grant to MJM from the Lindorf Family Foundation, Newark, OH. The funders had no role in study design, data collection and analysis, decision to publish, or preparation of the manuscript.

Copyright: (c) 2014 Middelveen MJ et al. This is an open access article distributed under the terms of the Creative Commons Attribution License, which permits unrestricted use, distribution, and reproduction in any medium, provided the original work is properly cited. Data associated with the article are available under the terms of the Creative Commons Zero "No rights reserved" data waiver (CC0 1.0 Public domain dedication).

How to cite this article: Middelveen MJ, Burke J, Sapi E et al. Culture and identification of Borrelia spirochetes in human vaginal and seminal secretions [version 1; peer review: 1 not approved] F1000Research 2014, 3:309

https://doi.org/10.12688/f1000research.5778.1

First published: 18 Dec 2014, 3:309 https://doi.org/10.12688/f1000research.5778.1 


\section{Introduction}

Lyme disease is the most common human tick-borne disease in the world today (Stricker \& Johnson, 2014). It is transmitted by Ixodes ticks and is caused by the spirochete Borrelia burgdorferi (Bb) (Burgdorfer et al., 1982). Bb is phylogenetically related to the spirochetal agent of syphilis, Treponema pallidum (Gupta et al., 2013). T. pallidum is transmitted sexually between partners through contact of mucosal membranes, gaining access to the bloodstream through microabrasions and then disseminating systemically (Ho \& Lukehart, 2011). The close phylogenic relationship of $\mathrm{Bb}$ to T. pallidum suggests that this mode of transmission might be possible for $\mathrm{Bb}$.

In addition to theoretical considerations, evidence for non-vector transmission of $\mathrm{Bb}$ is based on animal models. Proof of contact transmission of $\mathrm{Bb}$ - without involvement of an arthropod vector was established by two studies in mice. Burgess et al. (1986) caged uninfected deer mice with experimentally-infected deer mice and demonstrated transmission of $\mathrm{Bb}$ by seroconversion of contactexposed mice from negative to positive and by the isolation of $\mathrm{Bb}$ from the blood of one contact-exposed mouse 42 days after initial contact. A study by Wright \& Nielsen (1990) demonstrated that white-footed mice were susceptible to oral infection and transmitted infection to each other through direct contact. Furthermore, sexual transmission of $\mathrm{Bb}$ has been proposed in a canine model. $\mathrm{Bb}$ was transmitted to uninfected female dogs in estrus via semen by natural breeding with male dogs infected experimentally with $\mathrm{Bb}$ (Gustafson, 1993). Successful transmission of infection from male dogs to female dogs was shown by seroconversion of female dogs from negative to positive as well as the detection of $\mathrm{Bb}$ DNA in the tissue of fetuses from resulting pregnancies. If contact transmission of $\mathrm{Bb}$ occurs in mice and sexual transfer occurs in dogs, it is not unreasonable to postulate similar routes of infection in humans.

We sought to determine if viable Borrelia spirochetes could be recovered from human vaginal and seminal secretions, an important first step to investigate whether sexual transmission of these spirochetes among humans is possible.

\section{Materials and methods}

\section{Research subject selection}

Control subjects who were asymptomatic without a history of Lyme disease and patients with a history of Lyme disease were recruited for the study after written informed consent to collect and publish their data was obtained. Approval for sample collection was obtained from the Western Institutional Review Board, Olympia, WA (WIRB ${ }^{\circledR}$ \#20141439). Further approval for sample testing was obtained from the Institutional Review Board of the University of New Haven, West Haven, CT. Serological testing of all participants was performed by IGeneX Reference Laboratories, Palo Alto, CA. Patients were considered positive for Lyme disease if they were serologically positive or if they had musculoskeletal, neurocognitive or cardiac symptoms clinically consistent with a Lyme disease diagnosis. None of the patients were taking antibiotics at the time of testing.

\section{Borrelia cultures}

Borrelia spirochetes were cultured as previously described (Bankhead \& Chaconas, 2007; Middelveen et al., 2013b; Middelveen et al., 2014a). The inoculum for blood culture was prepared as follows: 10 milliliters of whole blood was collected by sterile venipuncture from each patient. Samples sat at room temperature for 10 to 15 minutes allowing clotting to occur. Red blood cells (RBCs) were separated by low speed centrifugation. Barbour-Stoner-Kelly $\mathrm{H}$ (BSK-H) complete medium was used for cultures with the addition of 6\% rabbit serum (Sigma Aldrich, \#B8291) and the following antibiotics: phosphomycin $(0.02 \mathrm{mg} / \mathrm{ml})$, rifampicin $(0.05 \mathrm{mg} / \mathrm{ml})$, and amphotericin B $(2.5 \mu \mathrm{g} / \mathrm{ml})$ (Sigma Aldrich).

The culture medium described above was inoculated for blood culture with the spun serum containing white blood cells and some RBCs, and for genital culture with either ejaculated semen or vaginal secretions collected by intravaginal swabbing with a sterile cotton-tipped swab. Blood and genital cultures were incubated at $32^{\circ} \mathrm{C}$ in an Oxoid anaerobic jar (Thermo Scientific) containing an AnaeroGen sachet (Thermo Scientific) to provide an anaerobic environment. Cultures were incubated for four weeks and checked weekly by light and/or darkfield microscopy for visible motile spirochetes.

All cultures were processed for microscopic imaging and PCR by centrifuging the culture fluid at $15,000 \mathrm{~g}$ for 20 minutes to concentrate spirochetes. The supernatant was discarded and the pellet retained.

\section{Dieterle silver staining}

Dieterle silver staining was performed using two fixation methods. In the standard method, formalin-fixed, paraffin-embedded pellets were sectioned and stained with Dieterle silver stain as previously described (Aberer \& Duray, 1991; Middelveen et al., 2013a). In the newer method, culture fluid was spread and dried on a SuperFrost ${ }^{\mathrm{TM}}$ Plus microscope slide (Fisher Scientific) and fixed by incubating the slide in acetone for 10 minutes at $-20^{\circ} \mathrm{C}$, as previously described (Sapi et al., 2013). Dieterle silver staining was performed on the acetone-fixed slide.

\section{Anti-Bb immunostaining}

A. McClain Laboratories. Blood and genital culture pellets were processed for special staining at McClain Laboratories LLC, Smithtown, NY. Formalin-fixed, paraffin-embedded pellets were sectioned and stained with Dieterle silver stain or anti-Bb immunostains for spirochete detection, as previously described (Middelveen et al., 2013a; Middelveen et al., 2014a). In brief, immunostaining was performed using an unconjugated rabbit anti-Bb polyclonal antibody (Abcam ab20950), incubated with an alkaline phosphatase probe (Biocare Medical \#UP536L), followed by a chromogen substrate (Biocare Medical \#FR805CHC), and counterstained with hematoxylin. Positive and negative controls were prepared for comparison purposes with liver sections from $\mathrm{Bb}$-inoculated mice and uninfected mice followed by Dieterle and immunostaining. Culture pellets from mixed Gram-positive bacteria and mixed Gram-negative bacteria were also prepared for comparison purposes as negative controls to exclude cross-reactivity with commonly encountered microorganisms. Staining was titrated to determine optimal antibody dilutions to achieve positive staining of spirochetes while minimizing background staining (Middelveen et al., 2013a; Middelveen et al., 2014a). 
B. University of New Haven. Samples were processed for $\mathrm{Bb}$ immunostaining as previously described (Sapi et al., 2013). Culture fluid was spread and dried on a SuperFrost ${ }^{\mathrm{TM}}$ Plus microscope slide (Fisher Scientific) and fixed by incubating the slide in acetone for 10 minutes at $-20^{\circ} \mathrm{C}$. Dried, fixed culture fluid was submerged under $100 \mu \mathrm{l}$ of polyclonal FITC-labeled rabbit anti-Bb antibody (Thermo Scientific \#PA-1-73005) diluted 1:50 in 1× PBS buffer with 1\% BSA (Sigma Aldrich \#A9418). For negative controls, the antibody was omitted and replaced with normal rabbit serum. The slides were then incubated for 1 hour at $37^{\circ} \mathrm{C}$ in a humidified chamber, washed with $1 \times$ PBS for 5 minutes at room temperature, rinsed twice in double distilled water and dried in a laminar air-flow hood for 10 minutes. The slides were mounted with Vectashield mounting medium with DAPI counterstain (Vector Labs) and viewed with fluorescent microscopy at $400 \times$ magnification with a Leica DM2500 microscope (Sapi et al., 2013).

\section{Molecular hybridization using Bb DNA probe}

The $\mathrm{Bb}$ molecular beacon DNA probe was generously provided by Dr. Alan MacDonald. Probe FlaB (sequence of 23 mer) was derived from the $\mathrm{Bb}$ open reading frame (ORF) BB0147 (approximately 1100 mer) of the flagellin B gene. A nucleotide Basic Local Alignment Search Tool (BLAST) search of the 23 mer sequence disclosed no matches in the human genome or in any other life form other than the Bb sequence of BB0147.

$\mathrm{Bb}$ detection with the molecular beacon was performed as previously described (Middelveen et al., 2014a) using the following protocol: paraffin sections were dewaxed by baking at $60^{\circ} \mathrm{C}$, then immersed in serial $100 \%$ xylene baths followed by serial immersion through baths of $100 \%$ ethanol, $90 \%$ ethanol, $80 \%$ ethanol, and finally in distilled $\mathrm{H}_{2} \mathrm{O}$, and then air-dried. Fixed sections were immersed in $20 \mu \mathrm{l}$ of the working DNA beacon solution. The sectioned specimen was covered with a layer of plastic cut from a Ziploc ${ }^{\circledR}$ freezer bag and was heated at $90^{\circ} \mathrm{C}$ for 10 minutes to denature DNA and RNA. The heat was first reduced to $80^{\circ} \mathrm{C}$ for 10 minutes, then the slides were removed from heat and allowed to gradually cool to $24^{\circ} \mathrm{C}$. The slides were washed in PBS, covered with $30 \%$ glycerol and a glass coverslip, then examined under an EPI Fluor microscope. Staining of test specimens was performed alongside staining of positive and negative controls. The positive control was prepared by embedding a known $\mathrm{Bb}$ strain in agarose, formalin-fixing the specimen then blocking in paraffin and staining sections as described above.

The specificity of the FlaB probe was validated in studies performed at the University of New Haven (Sapi E., unpublished observation 2014; see Supplemental Figure 1). The FlaB probe hybridized to Bb sensu stricto, yet failed to hybridize with B. afzelii, B. garinii, B. hermsii, Treponema denticola and Escherichia coli. Thus the probe appears to be specific for detection of Bb sensu stricto.

\section{PCR of cultures}

Blood and genital culture pellets were first dissolved in $200 \mu \mathrm{l}$ of Qiagen buffer, then forwarded to the University of New Haven, Department of Biology and Environmental Science, West Haven, CT, USA and Australian Biologics, Sydney, NSW, Australia for PCR detection of Borrelia.
A. Australian Biologics. Detection of Borrelia by PCR was performed as previously described (Mayne et al., 2012) using the $\mathrm{Eco}^{\mathrm{TM}}$ Real-Time PCR system with primers targeted to the genes encoding 16S rRNA (Borrelia), flA (T. denticola) and fliG1 (T. pallidum) and analyzed with the software version 3.0.16.0. DNA was extracted from the dissolved culture pellets using the QIAamp DNA Mini Kit (Qiagen) and $20 \mu \mathrm{l}$ were used for each reaction. The thermal profile involved incubation for 2 minutes at $50^{\circ} \mathrm{C}$, polymerase activation for 10 minutes at $95^{\circ} \mathrm{C}$ then PCR cycling for 40 cycles of 10 seconds at $95^{\circ} \mathrm{C}$ dropping to $60^{\circ} \mathrm{C}$ sustained for 45 seconds. All samples were run in duplicate with positive and negative controls. Positive controls were genomic DNA samples from B. burgdorferi, B. garinii, and B. afzelii (Amplirun DNA/RNA amplification controls, Vircell S.L, Granada, Spain). Negative controls were samples of non-template DNA in molecular-grade water. The magnitude of the PCR signal generated $(\Delta \mathrm{R})$ for each sample was interpreted as positive or negative compared to positive and negative controls.

In samples with sufficient DNA for sequencing, endpoint PCR amplification and Sanger sequencing of the Borrelia gene target from cultures was followed by BLAST comparison with known Borrelia sequences, as previously described (Mayne et al., 2012).

B. University of New Haven. DNA samples were extracted from blood, vaginal or seminal cultures by lysing cells overnight in $180 \mu \mathrm{l}$ tissue lysis buffer (Qiagen) and $20 \mu$ l Proteinase K (Qiagen) at $56^{\circ} \mathrm{C}$ in a shaking water bath followed by phenol:chloroform extraction the next day. The DNA was resuspended in 50-100 $\mu 11 \times \mathrm{TE}$ buffer.

A published TaqMan assay targeting a 139-bp fragment of the gene encoding the Borrelia 16S rRNA was used for the detection of Borrelia in DNA extracted from patient samples (O'Rourke et al., 2013). All reactions were carried out at a final volume of $20 \mu \mathrm{l}$ and consisted of $900 \mathrm{nM}$ of each primer, $200 \mathrm{nM}$ of probe, and $10 \mu \mathrm{l}$ of $2 \times$ TaqMan Universal PCR Master Mix (Applied Biosystems) and 1 nanogram of DNA. Amplifications were carried out on a CFX96 Real-Time System (Bio-Rad), and cycling conditions consisted of $50^{\circ} \mathrm{C}$ for 2 minutes, $95^{\circ} \mathrm{C}$ for 10 minutes, followed by 40 cycles of $95^{\circ} \mathrm{C}$ for 15 seconds and $60^{\circ} \mathrm{C}$ for 60 seconds. Fluorescent signals were recorded with CFX96 Real-Time software and Cq threshold was set automatically. The reactions were performed in triplicate with positive and negative controls.

Nested PCR primers for the genes encoding the Borrelia $16 \mathrm{~S}$ rRNA, fla and pyrG loci were used as previously described (Clark et al., 2013; Margos et al., 2010; Sapi et al., 2013). Reactions were carried out in a final volume of $50 \mu \mathrm{l}$ using $10 \mu \mathrm{l}$ template DNA. Final concentrations were $2 \times$ Buffer B (Promega), $2 \mathrm{mM} \mathrm{MgCl}$, $0.4 \mathrm{mM}$ dNTP mix, $2 \mu \mathrm{M}$ of each primer, and $2.5 \mathrm{U}$ Taq polymerase (Invitrogen). "Outer" primers were used in the first reaction. "Inner" primers were used for the nested reaction, in which $1 \mu \mathrm{l}$ of PCR product from the first reaction was used as template for the second. Cycling parameters were as follows: $94^{\circ} \mathrm{C}$ for 5 minutes followed by 40 cycles of denaturation at $94^{\circ} \mathrm{C}$ for 1 minute, annealing for 1 minute (temperature based on the primer set used), and 
extension at $72^{\circ} \mathrm{C}$ for 1 minute, with a final extension step at $72{ }^{\circ} \mathrm{C}$ for 5 minutes. PCR products were visualized on 1-2\% agarose gels. Sanger sequencing was used for gene analysis, as previously described (Margos et al., 2010).

\section{Results}

\section{Patient data}

All patient data are shown in Table 1. The control group included four asymptomatic patients (two males and two females). All four were seronegative for $\mathrm{Bb}$.

The patient group included six male subjects and seven female subjects, including four pairs of partners (Patients 6 and 7, 8 and 9, 10 and 11, and 12 and 13, respectively). Eleven of the 13 patients selected for the study were serologically positive for Lyme disease. Patient 1 was serologically equivocal and patient 8 was seronegative, although $\mathrm{Bb}$ plasmid DNA was detected in whole blood and serum from this patient.

\section{Light and darkfield microscopy}

Blood cultures from 11 patients were incubated for four weeks and checked weekly for spirochete growth using light and darkfield microscopy. Motile spirochetes and/or motile spherules were observed in the culture fluid from all 11 patients after four weeks (Table 2). Genital cultures from the four controls were incubated for four weeks. None of the control cultures contained visible spirochetes, and the cultures were sent for PCR testing. Genital cultures from the 11 patients were incubated for four weeks and checked weekly. Motile spirochetes were observed in the culture fluid from all 11 patients after four weeks (Figure 1A). See Dataset, data file 1 .

Most genital cultures grew very well and contained abundant spirochetes, but some blood cultures contained few spirochetes. Therefore, to better document the presence of spirochetes in culture, the culture fluid was concentrated into pellets by centrifugation (Table 3 ). Spirochetes and/or spherules were detected by sectioning and special staining of paraffin blocked pellets in all the patient blood and genital cultures concentrated by centrifugation, except for blood and genital culture pellets from Patient 1 that were lost during paraffin blocking (Table 3). Control genital culture samples were sent directly for PCR testing and were not subjected to light and darkfield microscopy.

\section{Immunohistochemistry}

A. Dieterle silver staining. Using standard Dieterle staining, spherules and/or spirochetal forms were visible in all patient genital cultures (Figure 1B). Spirochetes were detected in all patient genital culture pellets except for Patient 1, whose pellet was lost during processing (Table 3). Using the newer fixation method, spirochetes and sperm cells were visible in semen samples and showed distinct morphology (Figure 1C). Sperm cells are known to stain with silver stains (Pathak et al., 1979; Schmid et al., 1983). Sperm cells were seen in all semen samples except for Patients 2 and 6, who had vasectomies (data not shown). Since control genital cultures had no visible spirochetes, the control samples were sent directly for PCR testing and were not subjected to Dieterle silver staining. See Dataset, data file 2 .
Table 1. Patient Data. Patients $6 \& 7\left(^{*}\right), 8 \& 9$ $\left.{ }^{* *}\right), 10 \& 11(\dagger)$, and $12 \& 13(\dagger \dagger)$ are sexual partners. Patients 8 and 11 were seronegative but clinically diagnosed with Lyme disease.

\begin{tabular}{|l|l|l|l|}
\hline Control & Sex & Age & Serology \\
\hline $1(\mathrm{M})$ & male & 63 & negative \\
\hline $2(\mathrm{M})$ & male & 53 & negative \\
\hline $3(\mathrm{~F})$ & female & 58 & negative \\
\hline $4(\mathrm{~F})$ & female & 43 & negative \\
\hline
\end{tabular}

\begin{tabular}{|l|l|l|l|}
\hline Patient & Sex & Age & Serology \\
\hline $1(\mathrm{~F})$ & female & 56 & equivocal \\
\hline $2(\mathrm{M})$ & male & 45 & positive \\
\hline $3(\mathrm{M})$ & male & 35 & positive \\
\hline $4(\mathrm{~F})$ & female & 66 & positive \\
\hline $5(\mathrm{~F})$ & female & 27 & positive \\
\hline $6(\mathrm{M})^{\star}$ & male & 63 & positive \\
\hline $7(\mathrm{~F})^{\star}$ & female & 53 & positive \\
\hline $8(\mathrm{M})^{\star *}$ & male & 42 & negative \\
\hline $9(\mathrm{~F})^{\star *}$ & female & 40 & positive \\
\hline $10(\mathrm{M}) \dagger$ & male & 56 & positive \\
\hline $11(\mathrm{~F}) \dagger$ & female & 54 & negative \\
\hline $12(\mathrm{M}) \dagger \dagger$ & male & 65 & positive \\
\hline $13(\mathrm{~F}) \dagger \dagger$ & female & 54 & positive \\
\hline
\end{tabular}

Table 2. Microscopy results from fresh blood and genital culture fluid. See Dataset, data file 1. ND, not done.

\begin{tabular}{|c|c|c|}
\hline $\begin{array}{l}\text { Patient } \\
\text { number }\end{array}$ & $\begin{array}{l}\text { Microscopy - fresh } \\
\text { blood culture fluid }\end{array}$ & $\begin{array}{l}\text { Microscopy - fresh genital } \\
\text { culture fluid }\end{array}$ \\
\hline $1(F)$ & motile spherules & vaginal - motile spirochetes \\
\hline $2(M)$ & ND & seminal - ND \\
\hline $3(\mathrm{M})$ & ND & seminal - ND \\
\hline $4(F)$ & motile spherules & $\begin{array}{l}\text { vaginal - motile spherules and } \\
\text { spirochetes }\end{array}$ \\
\hline $5(F)$ & motile spherules & $\begin{array}{l}\text { vaginal - motile spirochetes, } \\
\text { some yeast cells }\end{array}$ \\
\hline $6(\mathrm{M})$ & $\begin{array}{l}\text { motile spirochetes } \\
\text { and spherules }\end{array}$ & seminal - motile spirochetes \\
\hline $7(F)$ & $\begin{array}{l}\text { motile spirochetes } \\
\text { and spherules }\end{array}$ & $\begin{array}{l}\text { vaginal - motile spherules and } \\
\text { spirochetes }\end{array}$ \\
\hline $8(\mathrm{M})$ & motile spherules & seminal - motile spirochetes \\
\hline $9(F)$ & motile spherules & vaginal - motile spirochetes \\
\hline $10(\mathrm{M})$ & motile spherules & $\begin{array}{l}\text { seminal - motile spherules/ } \\
\text { spirochetes }\end{array}$ \\
\hline $11(F)$ & motile spherules & $\begin{array}{l}\text { vaginal - motile spherules/ } \\
\text { spirochetes }\end{array}$ \\
\hline $12(\mathrm{M})$ & motile spherules & $\begin{array}{l}\text { seminal - motile spherules/ } \\
\text { spirochetes }\end{array}$ \\
\hline $13(F)$ & motile spherules & $\begin{array}{l}\text { vaginal - motile spherules/ } \\
\text { spirochetes }\end{array}$ \\
\hline
\end{tabular}




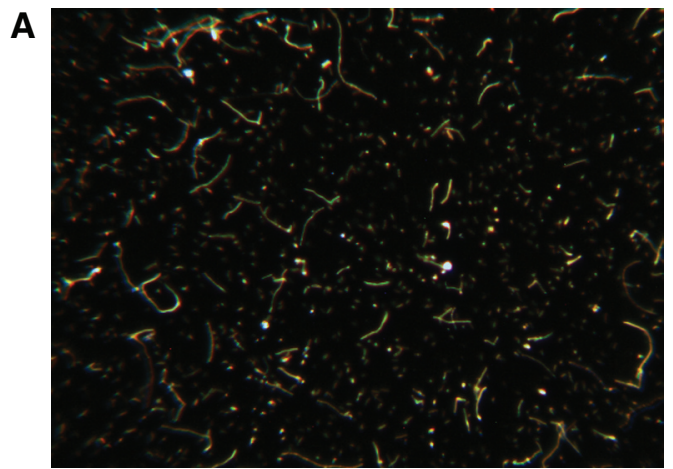

B
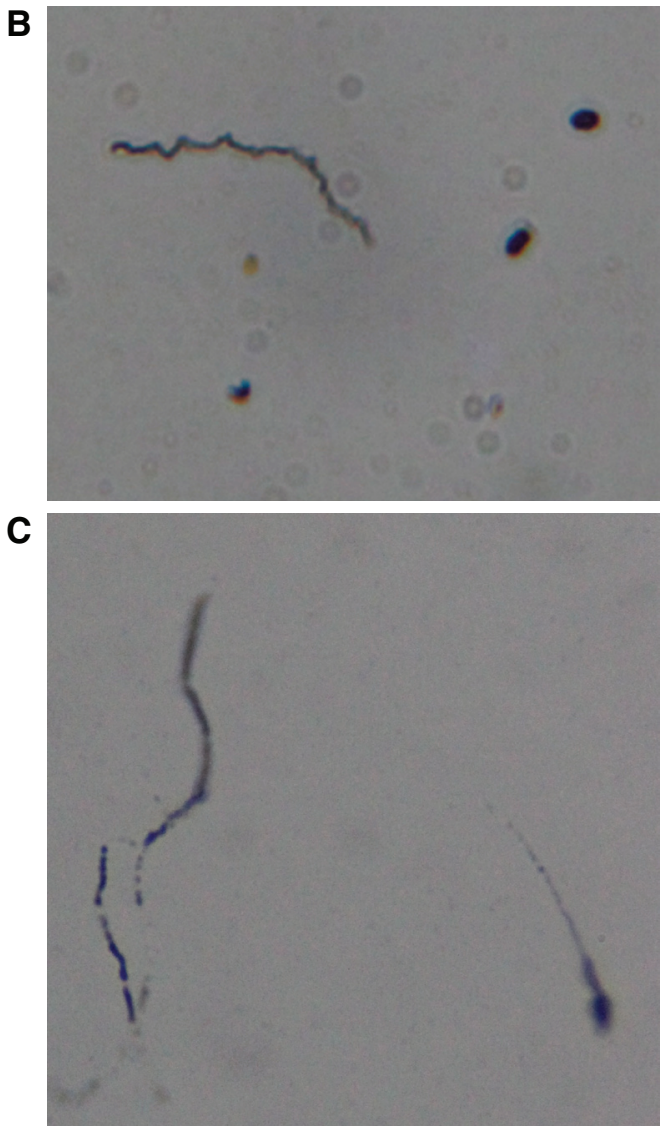

Figure 1. A: Darkfield image of genital culture from Patient 1. Note numerous spirochetes. 400× magnification. See Dataset, data file 1. B: Dieterle silver stain of genital culture from Patient 12. Note darkly staining spirochete. Formalin fixed slide, $400 x$ magnification. See Dataset, data file 2. C: Semen sample from Patient 10 showing B. burgdorferi spirochetes (left) and sperm cell (right). Dieterle silver stain of acetone fixed slide, 1000x magnification. See Dataset, data file 2.

\section{B. Anti-Bb immunostaining.}

\section{Culture fluid - University of New Haven}

Genital culture fluid from Patient 1 was fixed on a SuperFrost ${ }^{\mathrm{TM}}$ Plus microscope slide and was stained with FITC-labelled polyclonal anti-Bb antibody. Staining was strongly positive, revealing welldefined spirochetes morphologically consistent with Bb (Figure 2A). The polyclonal antibody was not reactive to T. denticola (data not shown).
Table 3. Microscopy results and Dieterle silver staining of genital culture concentrates. See Dataset, data file 2.

\begin{tabular}{|c|c|c|}
\hline $\begin{array}{l}\text { Patient } \\
\text { number }\end{array}$ & $\begin{array}{l}\text { Microscopy - genital } \\
\text { culture pellet }\end{array}$ & $\begin{array}{l}\text { Dieterle silver stain - } \\
\text { genital culture pellet }\end{array}$ \\
\hline $1(\mathrm{~F})$ & pellet lost & pellet lost \\
\hline $2(\mathrm{M})$ & $\begin{array}{l}\text { seminal - spherules/ } \\
\text { spirochetes }\end{array}$ & $\begin{array}{l}\text { seminal - spherules/ } \\
\text { spirochetes }\end{array}$ \\
\hline $3(\mathrm{M})$ & seminal - spirochetes & $\begin{array}{l}\text { seminal - spherules/ } \\
\text { spirochetes }\end{array}$ \\
\hline $4(F)$ & vaginal - spirochetes & vaginal - spherules \\
\hline $5(F)$ & $\begin{array}{l}\text { vaginal - spirochetes, } \\
\text { some yeast cells }\end{array}$ & vaginal - spherules \\
\hline $6(M)$ & seminal - spirochetes & $\begin{array}{l}\text { seminal - spherules/ } \\
\text { spirochetes }\end{array}$ \\
\hline $7(\mathrm{~F})$ & vaginal - spirochetes & $\begin{array}{l}\text { vaginal - spherules/ } \\
\text { spirochetes }\end{array}$ \\
\hline $8(\mathrm{M})$ & seminal - spirochetes & $\begin{array}{l}\text { seminal - spherules/ } \\
\text { spirochetes }\end{array}$ \\
\hline $9(F)$ & $\begin{array}{l}\text { vaginal - spherules/ } \\
\text { spirochetes }\end{array}$ & vaginal - spherules \\
\hline $10(\mathrm{M})$ & seminal - spirochetes & $\begin{array}{l}\text { seminal - spherules/ } \\
\text { spirochetes }\end{array}$ \\
\hline $11(F)$ & vaginal - spirochetes & $\begin{array}{l}\text { vaginal - spherules/ } \\
\text { spirochetes }\end{array}$ \\
\hline $12(\mathrm{M})$ & seminal - spirochetes & $\begin{array}{l}\text { seminal - spherules/ } \\
\text { spirochetes }\end{array}$ \\
\hline $13(F)$ & vaginal - spirochetes & $\begin{array}{l}\text { vaginal - spherules/ } \\
\text { spirochetes }\end{array}$ \\
\hline
\end{tabular}

\section{Culture pellets - McClain Laboratories}

Anti-Bb immunostaining was positive for all genital cultures except for Patient 1, whose pellet was lost during processing (Table 4). Immunostaining revealed both spiral and globular Bb forms (Figure 2B). Since control genital cultures had no visible spirochetes, the control samples were sent directly for PCR testing and were not subjected to immunostaining. See Dataset, data file 3.

\section{Molecular hybridization}

Hybridization with the Fla B probe was positive for genital culture pellets from Patients 2-9 (Table 4). The culture pellet from Patient 1 was lost during processing. The molecular probe showed intense staining in vaginal secretions and less intense staining in semen samples (Figure 3A and 3B). See Dataset, data file 4.

\section{PCR testing}

A. Australian Biologics. Borrelia 16S rRNA sequence was not detected by real-time PCR in any of the control genital culture pellets. In contrast, Borrelia 16S rRNA sequence was detected in genital culture pellets from 11 of 13 patients (Table 5A). Patient 2 had equivocal test results and Patient 3 had negative test results in seminal cultures. See Dataset, data file 5. Real-time PCR failed to detect treponemal gene sequences in any of the control or patient genital culture pellets. See Dataset, data file 5a. The 16S rRNA isolates from six patients were sequenced and subjected to BLAST analysis (see below). 
A

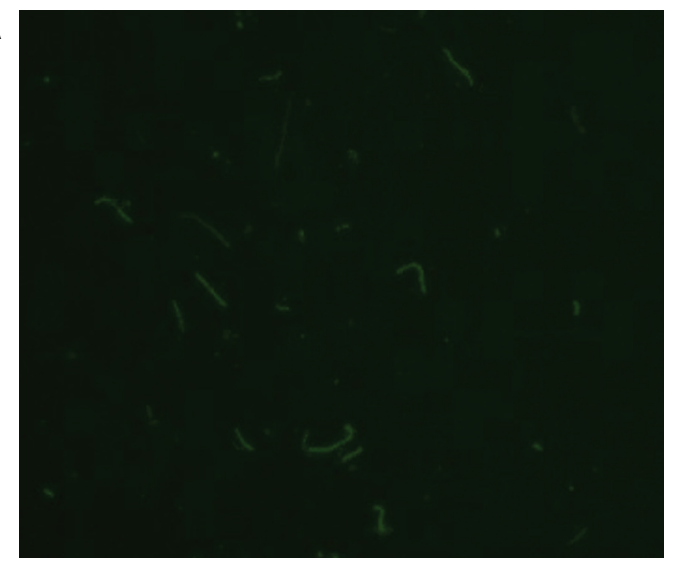

B

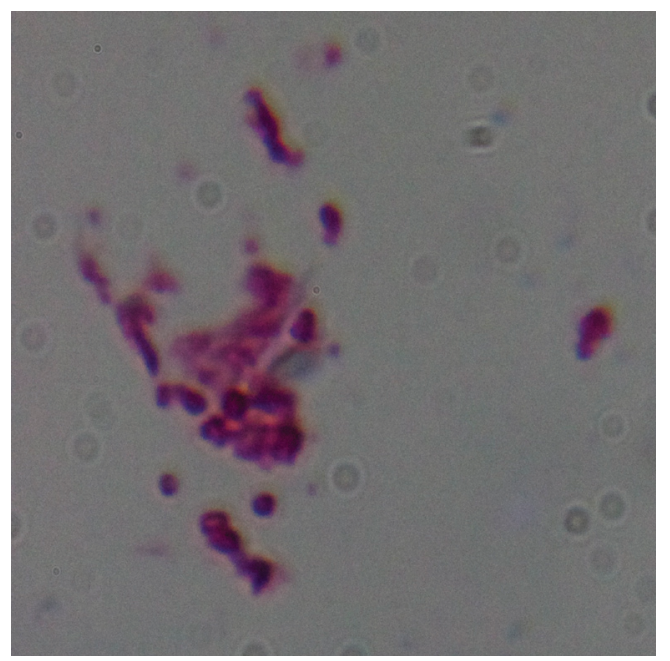

Figure 2. A: B. burgdorferi immunostaining of vaginal culture from Patient 1. Note intensely staining spiral and round forms in culture. $400 \times$ magnification. B: B. burgdorferi immunostaining of seminal culture from Patient 6 . Note intensely staining spiral and round forms in culture. 400x magnification. See Dataset, data file 3.

B. University of New Haven. PCR testing using the TaqMan assay for Borrelia 16S rRNA sequence was positive in blood culture pellets from seven of nine patients tested (Table 5B). Patients 1 and 5 had negative results in blood culture pellets using the TaqMan assay, but both were positive by nested PCR for the pyrG gene. In addition, nested PCR targeting the fla gene was performed on blood culture pellets from Patients 2, 3 and 4, and nested PCR targeting the 16S rRNA gene was performed on the blood culture pellet from Patient 6 . The samples were positive, and sequencing revealed 99-100\% homology with Bb sensu stricto strain B-31 (Table 5B). See Dataset, data file 7.

PCR testing using the TaqMan assay for Borrelia $16 \mathrm{~S}$ rRNA sequence was negative in all four control genital culture pellets, and nested PCR targeting the pyrG and fla genes was negative in all four control samples, confirming the results of the TaqMan assay (Table 5B). In contrast, eight of nine patients were positive for TaqMan 16S rRNA sequence in the genital culture pellets. Patient 6 was negative using the TaqMan assay for 16S rRNA sequence but positive

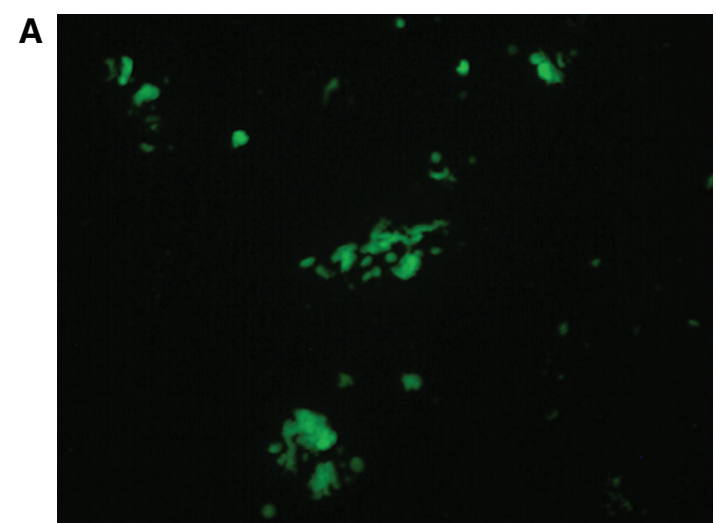

B

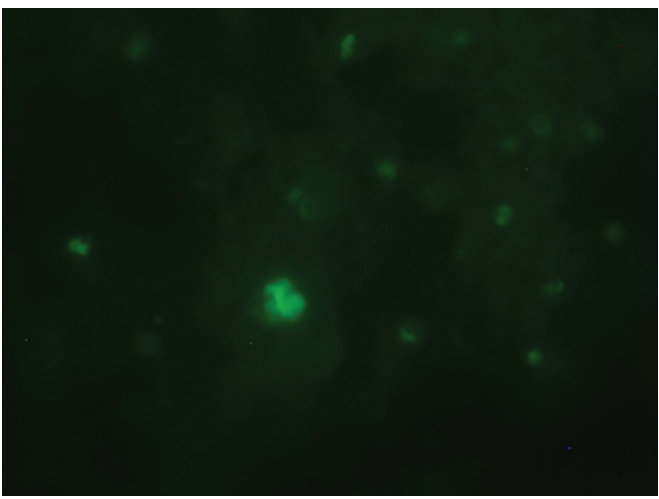

Figure 3. A: Molecular hybridization of B. burgdorferi-specific FlaB probe with seminal culture from Patient 6 . Note intensely staining spiral and round forms in culture. $400 \times$ magnification. B: Molecular hybridization of $B$. burgdorferi-specific FlaB probe with vaginal culture from Patient 7 . Note intensely staining spiral and round forms in culture. 400x magnification. See Dataset, data file 4.

\section{Table 4. Results of $B$. burgdorferi immunostaining} and FlaB molecular hybridization in genital culture concentrates. See Dataset, data files 3 and 4. ND, not done.

\begin{tabular}{|l|l|l|}
\hline $\begin{array}{l}\text { Patient } \\
\text { number }\end{array}$ & $\begin{array}{l}\text { Bb immunostaining - } \\
\text { genital culture pellet }\end{array}$ & $\begin{array}{l}\text { FlaB hybridization - } \\
\text { genital culture pellet }\end{array}$ \\
\hline $1(F)$ & pellet lost $^{*}$ & pellet lost \\
\hline $2(\mathrm{M})$ & seminal - positive & seminal - positive \\
\hline $3(\mathrm{M})$ & seminal - positive & seminal - positive \\
\hline $4(\mathrm{~F})$ & vaginal - positive & vaginal - positive \\
\hline $5(\mathrm{~F})$ & vaginal - positive & vaginal - positive \\
\hline $6(\mathrm{M})$ & seminal - positive & seminal - positive \\
\hline $7(\mathrm{~F})$ & vaginal - positive & vaginal - positive \\
\hline $8(\mathrm{M})$ & seminal - positive & seminal - positive \\
\hline $9(\mathrm{~F})$ & vaginal - positive & vaginal - positive \\
\hline $10(\mathrm{M})$ & seminal - positive & ND \\
\hline $11(\mathrm{~F})$ & vaginal - positive & ND \\
\hline $12(\mathrm{M})$ & seminal - positive & ND \\
\hline $13(\mathrm{~F})$ & vaginal - positive & ND \\
\hline
\end{tabular}

*Positive $\mathrm{Bb}$ immunostaining of genital culture fluid. See Results section. 
Table 5. A: Real time PCR testing of genital culture concentrates performed by Australian Biologics. ND, not done. See Dataset, data file 5. B: Real time and nested PCR testing of blood and genital culture concentrates performed by University of New Haven. See Dataset, data file 7. ND, not done.

Table 5A: Real-time PCR - Australian Biologics.

\begin{tabular}{|c|c|c|c|}
\hline $\begin{array}{l}\text { Control } \\
\text { number }\end{array}$ & $\begin{array}{l}\text { Genital culture - Real- } \\
\text { time Borrelia PCR }\end{array}$ & $\begin{array}{l}\text { Genital culture - Real- } \\
\text { time T. pallidum PCR }\end{array}$ & $\begin{array}{l}\text { Genital culture - Real- } \\
\text { time T. denticola PCR }\end{array}$ \\
\hline \multicolumn{4}{|l|}{$1(\mathrm{M})$} \\
\hline seminal & Negative & Negative & Negative \\
\hline \multicolumn{4}{|l|}{$2(M)$} \\
\hline seminal & Negative & Negative & Negative \\
\hline \multicolumn{4}{|l|}{$3(F)$} \\
\hline vaginal & Negative & Negative & Negative \\
\hline \multicolumn{4}{|l|}{$4(F)$} \\
\hline vaginal & Negative & Negative & Negative \\
\hline $\begin{array}{l}\text { Patient \# } \\
\text { sample }\end{array}$ & $\begin{array}{l}\text { Genital culture - Real- } \\
\text { time Borrelia PCR }\end{array}$ & $\begin{array}{l}\text { Genital culture - Real- } \\
\text { time T. pallidum PCR }\end{array}$ & $\begin{array}{l}\text { Genital culture - Real- } \\
\text { time T. denticola PCR }\end{array}$ \\
\hline $1(F)$ vaginal & $\begin{array}{l}\text { positive (sequenced } \\
\text { 99\% match B-31) }\end{array}$ & negative & negative \\
\hline $2(M)$ seminal & equivocal & negative & negative \\
\hline $3(\mathrm{M})$ seminal & negative & negative & negative \\
\hline $4(F)$ vaginal & positive & negative & negative \\
\hline $5(F)$ vaginal & positive & negative & negative \\
\hline $6(\mathrm{M})$ seminal & $\begin{array}{l}\text { positive (sequenced } \\
100 \% \text { match B-31) }\end{array}$ & negative & negative \\
\hline $7(F)$ vaginal & $\begin{array}{l}\text { positive (sequenced } \\
98 \% \text { match } \mathrm{B}-31 \text { ) }\end{array}$ & negative & negative \\
\hline $8(\mathrm{M})$ seminal & positive & negative & negative \\
\hline $9(F)$ vaginal & positive & negative & negative \\
\hline $10(\mathrm{M})$ seminal & $\begin{array}{l}\text { positive (sequenced } \\
100 \% \text { match YOR) }\end{array}$ & ND & ND \\
\hline $11(F)$ vaginal & $\begin{array}{l}\text { positive (sequenced } \\
100 \% \text { match YOR) }\end{array}$ & ND & ND \\
\hline \multicolumn{4}{|l|}{$12(\mathrm{M})$} \\
\hline seminal & positive & ND & ND \\
\hline \multicolumn{4}{|l|}{$13(F)$} \\
\hline vaginal & $\begin{array}{l}\text { positive (sequenced } \\
100 \% \text { match B-31) }\end{array}$ & ND & ND \\
\hline
\end{tabular}

Table 5B. PCR - University of New Haven.

\begin{tabular}{|l|l|l|l|}
\hline $\begin{array}{l}\text { Control } \\
\text { number }\end{array}$ & $\begin{array}{l}\text { Blood } \\
\text { culture }\end{array}$ & $\begin{array}{l}\text { Genital culture-16S } \\
\text { rRNA Taq Man PCR }\end{array}$ & Genital culture - Nested PCR \\
\hline $1(\mathrm{M})$ & ND & Negative & pyrG negative, fla negative \\
\hline $2(\mathrm{M})$ & ND & Negative & pyrG negative, fla negative \\
\hline $3(\mathrm{~F})$ & ND & Negative & pyrG negative, fla negative \\
\hline $4(\mathrm{~F})$ & ND & Negative & pyrG negative, fla negative \\
\hline
\end{tabular}




\begin{tabular}{|c|c|c|}
\hline Patient number & $\begin{array}{l}\text { Blood culture - primers } \\
\text { with positive detection }\end{array}$ & $\begin{array}{l}\text { Genital culture - primers } \\
\text { with positive detection }\end{array}$ \\
\hline $1(F)$ & pyrG & $16 S$ rRNA Taq Man \\
\hline $2(\mathrm{M})$ & $\begin{array}{l}\text { 16S rRNA Taq Man, } \\
\text { fla (sequenced, } 100 \% \\
\text { match B-31) }\end{array}$ & 16S rRNA Taq Man \\
\hline $3(\mathrm{M})$ & $\begin{array}{l}16 \text { S rRNA Taq Man, } \\
\text { fla (sequenced, } 100 \% \\
\text { match B-31) }\end{array}$ & $\begin{array}{l}\text { 16S rRNA Taq Man, } \\
\text { fla, } 16 \mathrm{~S} \text { rRNA (sequenced, } \\
99 \% \text { match B-31) }\end{array}$ \\
\hline $4(F)$ & $\begin{array}{l}16 \mathrm{~S} \text { rRNA Taq Man, } \\
\text { fla (sequenced, } 99 \% \\
\text { match B-31) }\end{array}$ & $16 S$ rRNA Taq Man \\
\hline $5(F)$ & pyrG & 16S rRNA Taq Man \\
\hline $6(\mathrm{M})$ & $\begin{array}{l}16 \mathrm{~S} \text { rRNA Taq Man } \\
16 \mathrm{~S} \text { rRNA (sequenced, } \\
99 \% \text { match B31) }\end{array}$ & $16 S$ rRNA \\
\hline $7(F)$ & 16S rRNA Taq Man, pyrG & $\begin{array}{l}16 S \text { rRNA Taq Man, } \\
16 S \text { rRNA, fla }\end{array}$ \\
\hline $8(\mathrm{M})$ & 16S rRNA Taq Man & 16S rRNA Taq Man \\
\hline $9(F)$ & 16S rRNA Taq Man & 16S rRNA Taq Man \\
\hline $10(\mathrm{M})$ & ND & ND \\
\hline $11(F)$ & ND & ND \\
\hline $12(\mathrm{M})$ & ND & $\begin{array}{l}\text { pyrG (sequenced, } 99 \% \\
\text { match B-31) }\end{array}$ \\
\hline $13(F)$ & ND & ND \\
\hline
\end{tabular}

using nested PCR targeting a different portion of the 16S rRNA gene (Table 5B). Nested PCR targeting the fla gene (Patient 3 ) and the 16S rRNA gene (Patients 3 and 7) was also performed on genital culture pellets and was positive in those patients, confirming the results of the TaqMan assay. Patient 12 had positive PCR targeting the pyrG gene with confirmatory sequencing (see below).

\section{Sequencing of Borrelia detected in blood and genital cultures}

PCR isolates of the vaginal culture from Patient 1 (Australian Biologics) and the seminal culture from Patient 3 (University of New Haven) were subjected to Sanger sequencing and BLAST analysis and showed 97-99\% homology with Bb sensu stricto strain B-31 (Table 5A and Table 5B). See Datasets, data files 6 and 7. PCR isolates of blood cultures from Patients 2, 3, 4 and 6 were subjected to Sanger sequencing and BLAST analysis at University of New Haven and showed 99-100\% homology with Bb sensu stricto strain B-31 (Table 5B). See Dataset, data file 7.

PCR isolates of genital cultures from three couples having unprotected sex (Patients 6-7, 10-11 and 12-13) were subjected to Sanger sequencing and BLAST analysis. Patients 6, 7, 10, 11 and 13 had sequencing done at Australian Biologics, while Patient 12 had sequencing done at University of New Haven. Sequencing revealed that the first and third couples had Borrelia strains that matched $\mathrm{Bb}$ sensu stricto strain B-31 (Table 6). In contrast, the second couple had PCR sequences that matched $B$. hermsii strain YOR. Thus the Borrelia strain shared by this couple differed significantly from the strains identified in the other couples. See Dataset, data file 6.
Dataset 1. Raw data of Borrelia spirochetes in human vaginal and seminal secretions

http://dx.doi.org/10.5256/f1000research.5778.d40491

The dataset contains data files 1, 2, 3, 4, 5, 5a, 6 and 7. Detailed legends for each files can be found in the text file provided.

\section{Discussion}

In this study using standard and published culture, immunohistochemical, molecular hybridization and PCR techniques, we have shown that Borrelia strains are present in semen and vaginal secretions from patients with Lyme disease. Simultaneous testing for treponemal spirochetes was negative in genital secretions of all Lyme disease patients, confirming the specificity of Borrelia detection in these patients. Furthermore we have shown that couples having unprotected sex have virtually identical strains of Borrelia in their genital secretions, suggesting that Borrelia spirochetes might be transmitted from person to person without a tick vector.

As expected, PCR sequencing of cultured Borrelia from semen and vaginal secretions yielded primarily $\mathrm{Bb}$ sensu stricto strains, reflecting the North American origin of our study subjects. In addition, PCR sequencing of genital secretions from one couple yielded identical strains of Bb sensu stricto strains in two different laboratories. However, we were surprised to find one couple with identical strains of $B$. hermsii in their genital secretions. The presence of a distinct Borrelia strain in semen and vaginal secretions from a sexually active couple that differs from strains found in other couples 
Table 6. Comparison of seminal and vaginal Borrelia gene sequences using BLAST

analysis. Sequencing for Patients 6, 7, 10, 11 and 13 was done at Australian Biologics.

Sequencing for Patient 12 was done at University of New Haven. See Dataset, data file 6.

\begin{tabular}{|l|l|l|l|l|l|l|}
\hline Patient & Description & $\begin{array}{l}\text { Maximum } \\
\text { Score }\end{array}$ & $\begin{array}{l}\text { Total } \\
\text { Score }\end{array}$ & $\begin{array}{l}\text { Query } \\
\text { Cover }\end{array}$ & E Value & $\begin{array}{l}\text { Reference } \\
\text { Strain Match }\end{array}$ \\
\hline $6(\mathrm{M})$ & Bb sensu stricto (B31) & 230 & 230 & $84 \%$ & $3 e-57$ & $100 \%$ \\
\hline $7($ F) & Bb sensu stricto (B31) & 224 & 224 & $83 \%$ & $2 e-55$ & $98 \%$ \\
\hline $10(M)$ & B. hermsii (YOR) & 32.2 & 1229 & $75 \%$ & 1.5 & $100 \%$ \\
\hline $11(F)$ & B. hermsii (YOR) & 30.2 & 599 & $84 \%$ & 2.1 & $100 \%$ \\
\hline $12($ M) & Bb sensu stricto (B31) & 1218 & 1218 & $95 \%$ & 1 e-63 & $99 \%$ \\
\hline $13(F)$ & Bb sensu stricto (B31) & 97.6 & 4880 & $87 \%$ & $1 e-20$ & $100 \%$ \\
\hline
\end{tabular}

supports the premise of Borrelia transmission via shared genital secretions. The finding is analogous to sharing distinct human immunodeficiency virus (HIV) strains, which is well recognized in sexual partners with HIV/AIDS (Shaw \& Hunter, 2012).

Animal models have provided compelling evidence for contact transmission of $\mathrm{Bb}$ without a tick vector in mice, ducks, cats and dogs (Burgess et al., 1986; Burgess \& Patrican, 1987; Burgess, 1989; Burgess, 1992; Wright \& Neilsen, 1990). Bb has been shown to survive in stored semen from dogs, rams and bulls (Kumi-Diaka \& Harris, 1995). Furthermore, seminal transmission of $\mathrm{Bb}$ has been noted in dogs, as described above (Gustafson, 1993). In contrast, contact transmission of $\mathrm{Bb}$ could not be demonstrated in Lewis rats and Syrian golden hamsters (Moody \& Barthold, 1991; Woodrum \& Oliver, 1999). Technical limitations in the study of these highly inbred rodents may have contributed to the negative results.

While it is not possible to perform controlled sexual transmission studies in humans, several investigators have speculated that this mode of transmission is possible (Bach, 2001; Harvey \& Salvato, 2003; Stricker et al., 2004). The suggestion that Bb could be transmitted sexually was initially proposed by Bach in 2001. He observed that sexually active patients had a marked propensity for antibiotic failure and speculated that re-infection occurred by intimate personto-person contact. Bb DNA was detected by PCR technology in human breast milk, umbilical cord blood, semen and vaginal secretions taken from patients presenting at his practice (Bach, 2001).

The study of a group of chronically ill Bb-seropositive and PCRpositive patients in Houston, Texas - a non-endemic area - provided epidemiological evidence that Lyme disease could spread in the absence of a suitable vector (Harvey \& Salvato, 2003). In the absence of infected ticks, intimate person-to-person transfer was implicated as the probable means of transmission (Harvey \& Salvato, 2003). A study by Stricker et al. provided clinical and immunological evidence for $\mathrm{Bb}$ transmission from partner to partner. In heterosexual seropositive couples with Lyme disease in which only one partner had a documented tick bite, the partner with the documented tick bite tended to have more severe clinical manifestations of the disease and a lower CD57 natural killer (NK) cell level (Stricker et al., 2004). This difference in clinical severity and CD57 NK cell level was not noted in seropositive couples diagnosed with Lyme disease in which both partners had a documented history of tick bite (Stricker et al., 2004). Sexual transfer of Borrelia infection through mucosal contact therefore seems possible in humans. The fact that we have been able to culture motile, actively reproducing, viable spirochetes from human genital secretions reinforces this hypothesis.

Recent reports from the Centers for Disease Control and Prevention (CDC) indicate that more than 300,000 cases of Lyme disease are diagnosed yearly in the USA (CDC, 2013). Sexual transmission of Borrelia may partly explain the large number of annual cases that is almost two times higher than breast cancer and six times higher that HIV/AIDS (Stricker \& Johnson, 2014). Recognition of possible sexual transmission of Borrelia in both humans and animals is fundamentally important because of the epidemiological implications. If sexual transmission of Borrelia occurs in both animals and humans, this mode of transmission is a possible means of introducing Borrelia infection into areas not considered endemic and of introducing the spirochete to new reservoirs. Borrelia would also join the list of other spirochetes that are either proven or postulated to be sexually transmitted, including the spirochetal agents of syphilis and leptospirosis (Harrison \& Fitzgerald, 1988; Maatouk \& Moutran, 2014).

Lyme disease diagnosis is based largely upon serological testing using CDC-sanctioned two-tier surveillance criteria supported by FDA-approved commercial test kits. While most patients in this study did have positive serological test results for Lyme borreliosis, some were considered serologically negative, and the majority of our study subjects did not meet the positive standard as defined by the CDC surveillance criteria (CDC, 2014a). We were able to detect Borrelia spirochetes in the blood and/or genital secretions of all patients who were clinically diagnosed with Lyme disease, demonstrating that the $\mathrm{CDC}$ surveillance protocol is inadequate diagnostically. Inadequate diagnostic methodology undoubtedly results in under-reporting of Lyme disease, and at least one group has speculated that this substandard methodology is considered acceptable because Borrelia is not sexually transmitted (Lange \& Sayyedi, 2002). In addition, if Borrelia spirochetes were transmitted sexually, then patients with false-negative results may unknowingly spread the infection to sexual partners.

The 2011 CDC case definition for Lyme disease states that a positive $\mathrm{Bb}$ culture confirms the diagnosis of the disease (CDC, 2014b). Although culture of Borrelia genital isolates may be a useful diagnostic laboratory methodology in the future, detecting and 
characterizing cultured Borrelia isolates is not straightforward, and both false-positive and false-negative results could occur. In our experience, human clinical isolates from genital secretions frequently propagate prolifically in culture, but on occasion they do not. In such instances, the culture must be concentrated and specific staining should be conducted to ascertain the presence of spirochetes. Once detected, spirochetes must be characterized genetically for specific identification. PCR is currently the most reliable means for correctly identifying cultured isolates, but even this methodology has drawbacks and limitations (Lange \& Sayyedi, 2002; Nolte, 2012).

There are currently no standardized FDA-approved PCR protocols or kits available for $\mathrm{Bb}$ detection, so commercial PCR testing constitutes an array of "home brew" assays using different methodologies such as real-time PCR and nested PCR, with various primers targeting different genes, yielding wide differences in sensitivity and specificity (Nolte, 2012; Schmidt, 1997; Yang et al., 2012). False negatives can result because primers may be strain-specific and may not detect all Borrelia genotypes, and fluids such as blood, semen and vaginal secretions may contain substances inhibitory to the PCR process (Lange \& Sayyedi, 2002; Nolte, 2012; Yang et al., 2012). The potential for false-positive PCR testing may also arise if there is DNA contamination in the laboratory, and appropriate positive and negative controls must be included in the assay (Nolte, 2012; Lange \& Sayyedi, 2002). We experienced differences in primer specificity in our clinical isolates and also found that inhibition occurred, particularly in semen cultures.

Another complicating factor in Borrelia isolation is the morphological variation of the spirochete, which includes spherical, granular or cystic forms. Morphological variants of $\mathrm{Bb}$, some of which are not culturable, are well documented in the medical literature (Barthold et al., 2010; Hodzic et al., 2014; Kurtti et al., 1987; Preac Mursic et al., 1996). These variants may play a role in infection, enabling $\mathrm{Bb}$ and other pathogenic spirochetes to evade the immune system (Döpfer et al., 2012; Menten-Dedoyart, 2012; Preac Mursic et al., 1996). Limited Bb growth and non-spiral morphology are thought to be induced by unfavorable environmental conditions (Brorson et al., 2009), and these features appear to be consistent with our observations. We found that Borrelia growth was more vigorous with more long slender morphological variants in cultures of genital secretions compared to cultures of blood, and we speculate that the human circulatory system is a more hostile environment for Borrelia than the human reproductive system.

Several questions have been raised about the likelihood of Borrelia sexual transmission (Craig, 2014). First, according to the CDC surveillance system Lyme disease occurs most commonly in children and older adults. However, the CDC surveillance system only captures about $10 \%$ of Lyme disease patients, and the other $90 \%$ may have a different demographic distribution consistent with sexual transmission, as shown in a recent study from Australia (Mayne, 2015). Second, while sexually transmitted diseases like herpes simplex virus (HSV) and gonorrhea show an urban predominance, Lyme disease has a more rural distribution (Craig, 2014). However, Lyme disease is acquired in more ways than HSV and gonorrhea, and the rate of sexual transmission is unknown at present. Thus the epidemiology of Lyme disease may differ from other sexually transmitted diseases based on these undefined variables. Third, the transmission of HIV can be traced from one sex partner to another using HIV strain typing. Based on our study, a similar transmission pattern using Borrelia strain typing may be seen once larger studies are performed among couples having unprotected sex. In summary, sexual transmission of Borrelia is plausible in light of our limited knowledge about the risk of acquiring Lyme disease.

In conclusion, we have shown that Borrelia spirochetes are present in semen and vaginal secretions of patients with Lyme disease. Furthermore, virtually identical strains of Borrelia are present in couples having unprotected sex, suggesting that transmission via intimate contact without a tick vector may occur. The epidemiology and clinical risk of Borrelia sexual transmission remain to be determined.

\section{Data availability}

F1000Research: Dataset 1. Raw data of Borrelia spirochetes in human vaginal and seminal secretions, 10.5256/f1000research.5778. d40491 (Middelveen et al., 2014b).

\section{Consent}

Written informed consent to publish clinical details and study results was obtained from each participant.

\section{Author contributions}

MJM recruited patients, performed the spirochete cultures and wrote the original manuscript. CB, KRF, AT and ES performed the IFA and PCR studies. JB, YW and AF performed the PCR studies. HAS and PJM provided patient samples and edited the manuscript. RBS recruited patients, coordinated all studies, revised the manuscript and edited it for publication. All authors approved the manuscript for publication.

\section{Competing interests}

The authors have no competing interests to declare. Preliminary results of the study were presented at the Western Regional Meeting of the American Federation for Medical Research, Carmel, CA, on January 25, 2014, and published in abstract form ( $J$ Invest Med 2014;62:280-1).

\section{Grant information}

Supported in part by a grant to MJM from the Lindorf Family Foundation, Newark, $\mathrm{OH}$. This work is dedicated to the memory of Dr. Willy Burgdorfer.

The funders had no role in study design, data collection and analysis, decision to publish, or preparation of the manuscript.

\section{Acknowledgements}

The authors thank Drs. Stewart Adams, Gordon Atkins, Robert Bransfield, George Chaconas, Douglas Demetrick, Dorte Dopfer, Christopher Hardy, Nick Harris, Doug Kahn, Alan MacDonald, Steve McClain, Kary Mullis, Jyotsna Shah, Leo Shea and Janet Sperling for helpful discussion. We are grateful to Dr. Robert B. Allan, Joel Israel and Anita Vieyra for technical support, and we thank Lorraine Johnson for manuscript review. 

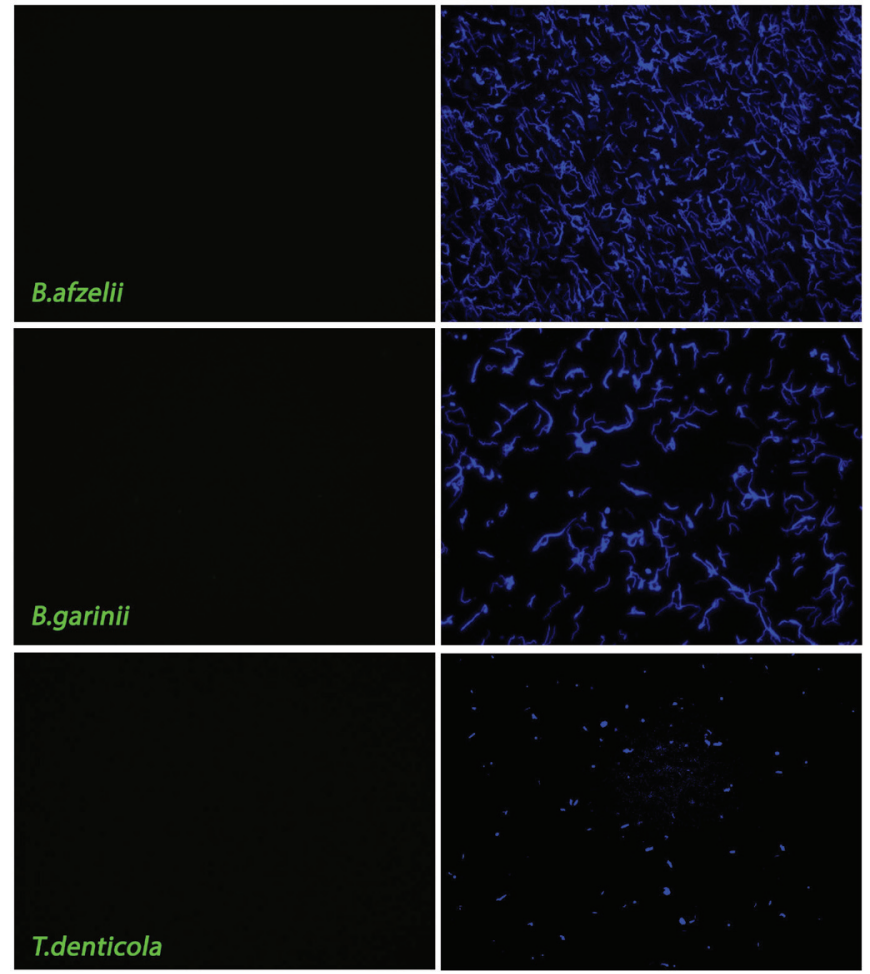
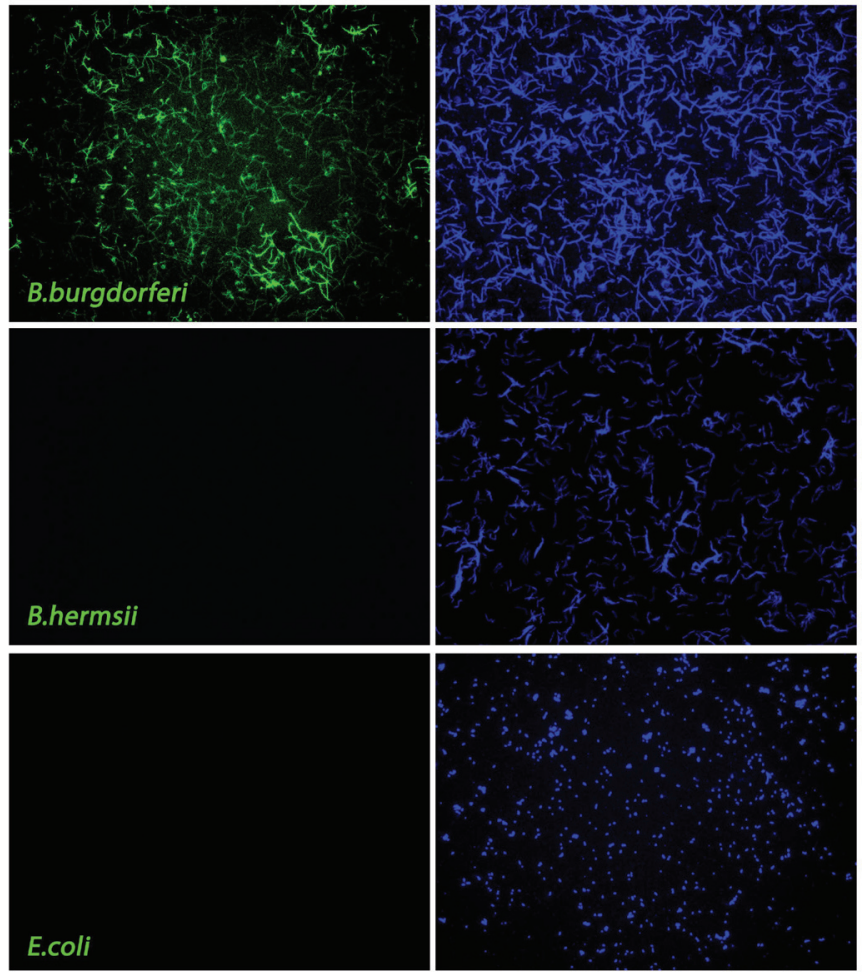

Supplemental figure 1. Molecular hybridization of FlaB probe with Borrelia strains, T. denticola and E.coli. FlaB hybridization is shown in green, while DAPI counterstain of bacterial targets is shown in blue. Note specific hybridization of FlaB probe with B. burgdorferi sensu stricto and lack of hybridization with other Borrelia strains, T. denticola or E.coli. 400x magnification. See Dataset, data file 4.

Aberer E, Duray PH: Morphology of Borrelia burgdorferi: structural patterns of cultured borreliae in relation to staining methods. J Clin Microbiol. 1991; 29(4): $764-72$

PubMed Abstract | Free Full Text

Bach G: Recovery of Lyme spirochetes by PCR in semen samples of previously diagnosed Lyme disease patients. International Scientific Conference on Lyme Disease. 2001.

Reference Source

Bankhead T, Chaconas G: The role of VIsE antigenic variation in the Lyme disease spirochete: persistence through a mechanism that differs from other pathogens. Mol Microbiol. 2007; 65(6): 1547-58

PubMed Abstract | Publisher Full Text

Barthold SW, Hodzic E, Imai DM, et al:: Ineffectiveness of tigecycline against persistent Borrelia burgdorferi. Antimicrob Agents Chemother. 2010; 54(2): $643-51$

PubMed Abstract | Publisher Full Text | Free Full Text

Brorson Ø, Brorson S, Scythes J, et al:: Destruction of spirochete Borrelia burgdorferi round-body propagules (RBs) by the antibiotic tigecycline. Proc Natl Acad Sci U S A. 2009; 106(44): 18656-61.

PubMed Abstract | Publisher Full Text | Free Full Text

Burgdorfer W, Barbour AG, Hayes SF, et al.: Lyme disease- a tick-borne spirochetosis? Science. 1982; 216(4552): 1317-9.

PubMed Abstract | Publisher Full Text

Burgess EC: Experimental inoculation of mallard ducks (Anas platyrhynchos platyrhynchos) with Borrelia burgdorferi. J Wildl Dis. 1989; 25(1): 99-102. PubMed Abstract | Publisher Full Text

Burgess EC: Experimentally induced infection of cats with Borrelia burgdorferi. Am J Vet Res. 1992; 53(9): 1507-11.

PubMed Abstract
Burgess EC, Amundson TE, Davis JP, et al: Experimental inoculation of Peromyscus spp. with Borrelia burgdorferi: evidence of contact transmission. Am J Trop Med Hyg. 1986; 35(2): 355-9.

PubMed Abstract

Burgess EC, Patrican LA: Oral infection of Peromyscus maniculatus with Borrelia burgdorferi and subsequent transmission by Ixodes dammini. Am J Trop Med Hyg. 1987; 36(2): 402-7.

PubMed Abstract

CDC 2013. Press Release: CDC provides estimate of Americans diagnosed with Lyme disease each year. 2013.

Reference Source

CDC: Lyme disease: Laboratory testing. 2014a.

Reference Source

CDC: Lyme disease: 2011 case definition. 2014b.

Reference Source

Clark KL, Leydet B, Hartman S: Lyme borreliosis in human patients in Florida and Georgia, USA. Int J Med Sci. 2013; 10(7): 915-31.

PubMed Abstract | Publisher Full Text | Free Full Tex

Craig E: Is Lyme disease an STD? Outside Magazine. 2014.

Reference Source

Döpfer D, Anklam K, Mikheil D, et al.: Growth curves and morphology of three

Treponema subtypes isolated from digital dermatitis in cattle. Vet J. 2012;

193(3): 685-93.

PubMed Abstract | Publisher Full Text

Gupta RS, Mahmood S, Adeolu S: A phylogenomic and molecular signature-based approach for characterization of the phylum Spirochaetes and its major clades: proposal for a taxonomic revision of the phylum. Front Microbiol. 2013; 4: 217. PubMed Abstract | Publisher Full Text | Free Full Text 
Gustafson JM: The in utero and seminal transmission of Borrelia burgdorferi in Canidae. 1993. PhD thesis, University of Wisconsin, Madison. 2014. Reference Source

Harrison NA, Fitzgerald WR: Leptospirosis--can it be a sexually transmitted disease? Postgrad Med J. 1988; 64(748): 163-4. PubMed Abstract | Publisher Full Text | Free Full Text Harvey WT, Salvato P: 'Lyme disease': ancient engine of an unrecognized borreliosis pandemic? Med Hypotheses. 2003; 60(5): 742-59. PubMed Abstract | Publisher Full Text

Ho EL, Lukehart SA: Syphilis: using modern approaches to understand an old disease. J Clin Invest. 2011; 121(12): 4584-92.

PubMed Abstract | Publisher Full Text | Free Full Text

Hodzic E, Imai D, Feng S, et al:: Resurgence of persisting non-cultivable Borrelia burgdorferi following antibiotic treatment in mice. PLOS One. 2014; 9(1): e86907.

PubMed Abstract | Publisher Full Text | Free Full Text

Kumi-Diaka J, Harris O: Viability of Borrelia burgdorferi in stored semen. $\mathrm{Br}$ Vet J. 1995; 151(2): 221-4.

PubMed Abstract | Publisher Full Text

Kurtti TJ, Munderloh UG, Johnson RC, et al.: Colony formation and morphology in Borrelia burgdorferi. J Clin Microbiol. 1987; 25(11): 2054-8.

PubMed Abstract | Free Full Text

Lange R, Sayyedi S: Evidence of Lyme borreliosis infection from the viewpoint of laboratory medicine. Int J Med Microbiol. 2002; 291(Suppl 33): 120-4.

PubMed Abstract | Publisher Full Text

Maatouk I, Moutran R: History of syphilis: between poetry and medicine. J Sex Med. 2014; 11(1): 307-10.

PubMed Abstract | Publisher Full Tex

Margos G, Hojgaard A, Lane RS, et al.: Multilocus sequence analysis of Borrelia bissettii strains from North America reveals a new Borrelia species, Borrelia

kurtenbachii. Ticks Tick Borne Dis. 2010; 1(4): 151-8.

PubMed Abstract | Publisher Full Text | Free Full Text

Mayne PJ: Investigations of Borrelia burgdorferi genotypes in Australia obtained from erythema migrans tissue. Clin Cosmet Investig Dermatol. 2012; 5: 69-78. PubMed Abstract | Publisher Full Text | Free Full Text

Mayne PJ: Clinical determinants of Lyme borreliosis, babesiosis, bartonellosis, anaplasmosis and ehrlichiosis in an Australian cohort. Int J Gen Med. 2015; 8: $1-12$.

Menten-Dedoyart C, Faccinetto C, Golovchenko M, et al.: Neutrophil extracellular traps entrap and kill Borrelia burgdorferi sensu stricto spirochetes and are not affected by Ixodes ricinus tick saliva. J Immunol. 2012; 189(11): 5393-5401. PubMed Abstract | Publisher Full Text

Middelveen MJ, Mayne PJ, Kahn DG, et al.: Characterization and evolution of dermal filaments from patients with Morgellons disease. Clin Cosmet Investig Dermatol. 2013a: 6: 1-21.

PubMed Abstract | Publisher Full Text | Free Full Text

Middelveen MJ, Burugu D, Poruri A, et al.: Association of spirochetal infection with Morgellons disease [v1; ref status: indexed, http://f1000r.es/8g] F1000Res. 2013b; 2: 25

PubMed Abstract | Publisher Full Text | Free Full Text

Middelveen MJ, McClain SA, Bandoski C, et al.: Granulomatous hepatitis

associated with chronic Borrelia burgdorferi infection: a case report. Res Open
Access. 2014a; 1: 875

Publisher Full Text

Middelveen MJ, Burke J, Sapi E, et al:: Raw data of Borrelia spirochetes in

human vaginal and seminal secretions. F1000Research. 2014b.

Data Source

Moody KD, Barthold SW: Relative infectivity of Borrelia burgdorferi in Lewis rats by various routes of inoculation. Amer J Trop Med Hyg. 1991; 44(2): 135-9.

PubMed Abstract

Mursic VP, Wanner G, Reinhardt S, et al:: Formation and cultivation of Borrelia burgdorferi spheroplast-L-form variants. Infection. 1996; 24(3): 218-26.

PublMed Abstract | Publisher Full Text

Nolte O: Nucleic Acid Amplification Based Diagnostic of Lyme (Neuro-) borreliosis - Lost in the Jungle of Methods, Targets, and Assays? Open Neurol 2012: 6(Suppl 1-M7): 129-139.

PubMed Abstract | Publisher Full Text | Free Full Text

O'Rourke M, Traweger A, Lusa L, et al.: Quantitative detection of Borrelia burgdorferi sensu lato in erythema migrans skin lesions using internally controlled duplex real time PCR. PLoS One. 2013; 8(5): e63968.

PubMed Abstract | Publisher Full Text | Free Full Text

Pathak S, Lau YF, Drwinga HL: Observations on the synaptonemal complex in Armenian hamster spermatocytes by light microscopy. Chromosoma. 1979; 73(1): 53-60.

PubMed Abstract | Publisher Full Tex

Sapi E, Pabbati N, Datar A, et al: Improved culture conditions for the growth and detection of Borrelia from human serum. Int J Med Sci. 2013; 10(4): 362-76. PubMed Abstract | Publisher Full Text | Free Full Text

Schmidt BL: PCR in laboratory diagnosis of human Borrelia burgdorfer infections. Clin Microbiol Rev. 1997; 10(1): 185-201.

PubMed Abstract | Free Full Text

Schmid M, Müller H, Stasch S, et al.: Silver staining of nucleolus organizer regions during human spermatogenesis. Hum Genet. 1983; 64(4): 363-70. PubMed Abstract | Publisher Full Text

Shaw GM, Hunter E: HIV transmission. Cold Spring Harb Perspect Med. 2012 2(11): a006965.

PubMed Abstract | Publisher Full Text | Free Full Text

Stricker RB, Johnson L: Lyme disease: Call for a "Manhattan Project" to combat the epidemic. PLoS Pathog. 2014; 10(1): e1003796.

PubMed Abstract | Publisher Full Text | Free Full Tex

Stricker RB, Moore DH, Winger EE: Clinical and immunologic evidence for transmission of Lyme disease through intimate human contact. $J$ Investig Med. 2004; 52: S151.

Woodrum JE, Oliver JH Jr: Investigation of venereal, transplacental, and contact transmission of the Lyme disease spirochete, Borrelia burgdorferi, in Syrian

hamsters. J Parasitol. 1999; 85(3): 426-30.

PubMed Abstract | Publisher Full Text

Wright SD, Nielsen SW: Experimental infection of the white-footed mouse with Borrelia burgdorferi. Am J Vet Res. 1990; 51(12): 1980-7.

PubMed Abstract

Yang J, Liu Z, Guan G, et al.: Evaluation of molecular methods for detection of Borrelia burgdorferi senso lato in ticks. Diagn Microbiol Infect Dis. 2012; 73(1): 80-3.

PubMed Abstract | Publisher Full Text 


\section{Open Peer Review}

\section{Current Peer Review Status: $\mathrm{X}$}

\section{Version 1}

Reviewer Report 05 January 2015

https://doi.org/10.5256/f1000research.6178.r7090

(C) 2015 Donta S. This is an open access peer review report distributed under the terms of the Creative Commons Attribution License, which permits unrestricted use, distribution, and reproduction in any medium, provided the original work is properly cited.

\section{Sam T. Donta}

Falmouth Hospital, Falmouth, MA, USA

There are a number of issues that mitigate against the authors' conclusion that Lyme disease can be transmitted sexually.

While there are conflicting reports from animal studies that there can be transmission by contact between animals and other studies that appear to better controlled that do not provide such evidence, there is no obvious epidemiological evidence in humans that this is a likely possibility. Apart from the sociologic implications of claiming that intimate or even casual transmission is possible, there needs to be more compelling evidence that this might be the case than is offered in this report.

Specific issues in this report:

1. No evidence that samples were blinded.

2. The numbers of patients were too small from which to draw meaningful conclusions.

3. The actual serologic data on patients should be presented in order to be more properly assessed.

4. PCR-DNA analyses should have been done on the original specimens; again in a blinded fashion, and weekly during the culture period. And, as PCR-DNA testing is much more sensitive than cultures, without this information, the validity of the presented information remains in question.

5. Concurrent samples of other body fluids, i.e. blood, saliva, should have been included.

6. Even if presuming the presence of the Lyme borrelia in vaginal secretions or semen, the numbers present would have not been sufficient to cause any transmission of infection, as, with any infectious process, there needs to be a critical inoculum to establish infection. This is the case with transmission by ticks. 
7. Borrelia may be spirochetes as are Treponemes and Leptospira, but the transmission of the latter are obviously through body fluids, and not by tick or other vectors. It is not clear that infections transmitted by ticks are also transmitted by intimate or casual contact.

8. If it was true that $8 / 11$ samples were positive, one would expect a much more obvious clinical picture of transmission by intimate or casual contact, which is not the case.

9. PCR-DNA analyses and long-term cultures can be subject to contamination, making the data here more difficult to interpret.

Competing Interests: No competing interests were disclosed.

\section{I confirm that I have read this submission and believe that I have an appropriate level of expertise to state that I do not consider it to be of an acceptable scientific standard, for reasons outlined above.}

Author Response 12 Jan 2015

Raphael Stricker, International Lyme and Associated Diseases Society, Bethesda, USA

Co-written with Marianne J. Middelveen

There are a number of issues that mitigate against the authors' conclusion that Lyme disease can be transmitted sexually.

We did not conclude that "Lyme disease can be transmitted sexually". Based on preliminary editorial comments, we were careful to state that our microscopy, immunochemistry, molecular hybridization and PCR analysis showing live, culturable Borrelia in semen and vaginal secretions suggests that these spirochetes could be transmitted in that manner. Our study does not prove this form of transmission, and we do not make this claim anywhere in the text.

While there are conflicting reports from animal studies that there can be transmission by contact between animals and other studies that appear to better controlled that do not provide such evidence, there is no obvious epidemiological evidence in humans that this is a likely possibility. Apart from the sociologic implications of claiming that intimate or even casual transmission is possible, there needs to be more compelling evidence that this might be the case than is offered in this report.

The statement that "better controlled" studies do not provide support for contact transmission of Borrelia in animals is contrary to the examples in mice and dogs described in the Introduction and Discussion sections of our article. We have pointed out that the two studies on highly inbred rodents that allegedly showed lack of such transmission did not use PCR techniques and therefore may have missed this transmission. The fact that the CDC now admits to more than 300,000 new cases of Lyme disease each year in the USA (and perhaps as many as one million new cases, as outlined in Stricker \& Johnson, 2014) is suggestive that other forms of transmission occur, as noted in the Discussion section on page 10. 


\section{No evidence that samples were blinded.}

In response to the referee's comment, we have noted that the laboratory testing was performed on coded samples in a blinded fashion. This has been noted in the Abstract and reiterated throughout the Methods section.

2. The numbers of patients were too small from which to draw meaningful conclusions.

The "meaningful conclusions" are that microscopy, immunochemistry, molecular hybridization and PCR analysis demonstrates live, culturable Borrelia in semen and vaginal secretions from Lyme disease patients. Although the numbers are relatively small, we feel that our detailed study supports this conclusion.

3.The actual serologic data on patients should be presented in order to be more properly assessed.

We can include the actual serologic data as an original Dataset, but we don't see how that would alter the experimental findings in our study. The serologic data is presented in Table 1 and the Results section. In response to the referee's comment, we have added more detail about the serologic and diagnostic criteria in the Methods section on page 4 , with supporting references.

4. PCR-DNA analyses should have been done on the original specimens; again in a blinded fashion, and weekly during the culture period. And, as PCR-DNA testing is much more sensitive than cultures, without this information, the validity of the presented information remains in question.

PCR-DNA analysis was done on the "original specimens" in a blinded fashion in conjunction with the microscopy, immunochemistry and molecular hybridization analysis. Repeated testing at weekly or other intervals is beyond the scope of this pilot study, and this type of testing should certainly be explored in future Lyme disease studies.

5. Concurrent samples of other body fluids, i.e. blood, saliva, should have been included.

As shown in Table 5, we did do PCR testing on concurrent blood samples in some patients. Saliva testing for Borrelia would have been of interest, but this form of Borrelia testing requires further investigation and is beyond the scope of our study.

6. Even if presuming the presence of the Lyme borrelia in vaginal secretions or semen, the numbers present would have not been sufficient to cause any transmission of infection, as, with any infectious process, there needs to be a critical inoculum to establish infection. This is the case with transmission by ticks.

In response to the referee's comment, we have researched the number of spirochetes necessary for transmission of $B$. burgdorferi infection in mice and $T$. 
pallidum infection in humans. The results show that a very small number of spirochetes (as little as 18 organisms) are required for transmission of infection, and we have included this information in the Discussion on page 11. We have also noted that seminal plasma and the female genital tract may provide a relatively permissive environment for spirochetes compared to blood, skin and other immune sites, making transmission theoretically easier via the genital route.

Borrelia may be spirochetes as are Treponemes and Leptospira, but the transmission of the latter are obviously through body fluids, and not by tick or other vectors. It is not clear that infections transmitted by ticks are also transmitted by intimate or casual contact.

In response to the referee's query, we have provided examples of other agents ( Babesia, Chlamydia, Coxiella) that are proven or postulated to be transmitted by both tickbite and intimate contact. This information is included in the Discussion on page 11 with supporting references.

If it was true that 8/11 samples were positive, one would expect a much more obvious clinical picture of transmission by intimate or casual contact, which is not the case.

As noted above, the substantial numbers of new Lyme disease cases each year suggests additional forms of transmission beyond a tickbite. At this time, the true epidemiology of Lyme disease is unknown because the CDC surveillance system only captures less than $10 \%$ of Lyme disease cases, as noted in the Discussion on page 11. Other epidemiological studies have suggested that some infected patients may be relatively asymptomatic (Harvey \& Salvato, 2003), so transmission via intimate contact resulting in less obvious infection is plausible. The risk of this form of transmission and correlation with symptoms merits further study. Our report simply raises the possibility, and rejecting the report will shove this issue under the rug to the detriment of Lyme patients.

9. PCR-DNA analyses and long-term cultures can be subject to contamination, making the data here more difficult to interpret.

PCR-DNA analysis is subject to contamination. That is why we did blinded testing that always included negative controls in three different laboratories using microscopy, immunochemistry and molecular hybridization to confirm the PCR findings. Although PCR testing alone might be "difficult to interpret", the combination of experimental techniques done in different laboratories makes interpretation much more reliable. We hope that the referee will change his opinion after reading the revised manuscript and our responses to his comments.

Competing Interests: No competing interests were disclosed. 


\section{Comments on this article}

\section{Version 1}

Reader Comment 21 Jan 2015

Phillip Baker, American Lyme Disease Foundation, USA

Since the optimal $\mathrm{pH}$ for the growth of Borrelia burgdorferi is $\mathrm{pH} 7.6$ and the $\mathrm{pH}$ of vaginal secretions is quite acidic, ranging from pH $3.8-\mathrm{pH} 4.5$, perhaps Dr. Stricker would like to offer an explanation as to how long -- if at all-- B. burgdorferi is likely to survive in such a "hostile" environment.

Competing Interests: None

Reader Comment 19 Jan 2015

Sin Hang Lee, Milford Molecular Diagnostics, USA

Dr. Baker's comment linking Koch's postulates to the CDC Lyme disease case criteria which is based on the two-tier serology test results is not appropriate. The Koch's postulates ${ }^{1}$ were published before the discovery of antibodies. In fact, attempts to rely on the Widal test (an antibody test) to diagnose typhoid fever were rejected more than 100 years ago ${ }^{2}$. A CDC document clearly states that serology tests are not reliable for the diagnosis of typhoid fever ${ }^{3}$. If the Koch's postulates are to be applied in the diagnosis of Lyme disease, alternative methods, e.g. DNA sequencing-based tests $^{4}$, to detect causative agents must be used.

\section{References}

1. Koch R: Untersuchungen Über die Aetiologie der Wundinfectionskrankheiten. 1878; Leipzig: F.C.W. Vogel.

2. Jürgens GJ: Zur Ätiologie und Pathogenese des Abdominaltyphus. 1904; Ztschr. f. klin. Med., Berl..

3. Newton AE, Mintz E: Chapter 3 Infectious Diseases Related To Travel: Typhoid \& Paratyphoid Fever In CDC Health Information for International Travel. CDC. August 01, 2013. Reference Source 4. Lee SH, Vigliotti JS, Vigliotti VS, Jones W, et al.: DNA sequencing diagnosis of off-season spirochetemia with low bacterial density in Borrelia burgdorferi and Borrelia miyamotoi infections. Int J Mol Sci. 2014; 15 (7): 11364-11386 PubMed Abstract | Publisher Full Text

Competing Interests: Competing Interests: Dr. Sin Hang Lee is the director of Milford Molecular Diagnostics, a CLIA-certified laboratory offering DNA sequencing-based tests for Lyme and related borrelioses 
Phillip Baker, American Lyme Disease Foundation, USA

Despite Dr. Stricker's attempts to obfuscate the key issues, the fact remains -- as noted by Donta in his review - that the mere presence of Borrelia in seminal and vaginal secretions, if that finding indeed can be confirmed independently by other investigators, does not prove that they cause disease, especially in view of the fact that most of the patients examined were not seropositive by standard CDC test criteria. Perhaps Dr. Stricker ought to review Koch's postulates for guidance in that regard. In the absence of such proof, it is irresponsible and not in the best interest of the public health for Dr. Stricker to alarm the public by touting the results of preliminary and unconfirmed findings in the press.

Most recent data from the CDC still continue to show that, unlike the reported incidence of sexually transmitted diseases, reported cases of Lyme disease are concentrated in the same 13 States as in previous years. Obviously, the epidemiology is the same whether the reported number of cases is $30 \mathrm{~K}$ or $300 \mathrm{~K}$ per year.

Competing Interests: No competing interests were disclosed.

Author Response 03 Jan 2015

Raphael Stricker, International Lyme and Associated Diseases Society, Bethesda, USA

Co-written with Marianne J. Middelveen

We appreciate Phillip Baker's interest in our study showing live, culturable Borrelia spirochetes in semen and vaginal secretions from patients with Lyme disease. We used microscopy, immunochemistry, molecular hybridization and polymerase chain reaction (PCR) techniques to demonstrate the presence of these live spirochetes in cultures of genital secretions, and as stated in the study our results suggest that Lyme disease could be sexually transmitted. All of the concerns in Baker's commentary were previously aired on the Internet, and these concerns were addressed in our report. His objection has little merit for the reasons described below.

Baker represents a group of researchers who cling to the concept that Lyme disease is a trivial illness that is "hard to catch and easy to cure", and that chronic Lyme disease due to persistent infection with the Lyme spirochete, Borrelia burgdorferi, is rare or non-existent ${ }^{1,2}$. As discussed elsewhere, this "Lyme denialist" philosophy and disregard for opposing viewpoints has been a major factor in the epidemic spread of Lyme disease, which accounts for more than 300,000 new cases per year in the USA according to the latest Centers for Disease Control and Prevention (CDC) estimates ${ }^{2}$. As stated in our article, this alarming epidemic is twice as common as breast cancer and six times more common than HIV/AIDS, and its prevalence is hard to explain by tickbite transmission alone. Thus from an epidemiological perspective, sexual transmission of the live Borrelia spirochetes found in genital secretions is a plausible mechanism for the spread of Lyme disease.

Baker's commentary on our article uses selective information that supports his point of view while 
ignoring contradictory evidence. He starts with two animal models that appear to refute contact or sexual transmission of Lyme disease, and he concludes with selective epidemiological evidence from the CDC that obliquely supports his view. Sandwiched in between is a flawed discussion of maternal-fetal transmission of Lyme disease that has no bearing on sexual transmission of the spirochetal infection. Although we have addressed the animal model and epidemiological issues in our article, we will address these issues in greater detail below. We will leave it to readers to find the flaws in Baker's denial of maternal-fetal transmission of Lyme disease, since this form of transmission has been documented in canine, bovine, murine and human reports from the peerreviewed medical literature ${ }^{3-8}$.

Baker cites two animal studies as proof that Lyme disease cannot be sexually transmitted ${ }^{9,10}$. The study by Moody and Barthold was performed using Lewis rats, while the study by Woodrum and Oliver was performed using Syrian hamsters. In limiting his analysis to these two studies, Baker ignores the compelling observations in mice, birds, cats and dogs that support contact or sexual transmission of Lyme disease without a tick vector, as outlined in our report ${ }^{4,11-13}$. The Lewis rat and Syrian hamster studies had significant flaws. First, these rodents are highly inbred with all Lewis rats descended from a single breeding colony, and all Syrian hamsters descended from a single female breeder ${ }^{14,15}$. The effect of inbreeding on contact transmission of Borrelia is unknown. Second, these rodents are poor models for human Lyme disease. The Lewis rat develops a transient arthritis that fades without treatment following intraperitoneal injection of Borrelia, while the Syrian hamster may be colonized by Borrelia for long periods without showing any signs of disease ${ }^{16,17}$. Since the disease pattern in these animals differs significantly from the human pattern, it is not surprising that transmission of Borrelia would differ as well. In fact, the immunocompetent Syrian hamster has been abandoned as a model for human Lyme disease because of these differences. Third and perhaps most important, both studies used limited culture and immunological methods to determine sexual transmission of Borrelia, and more sensitive detection of spirochetes by molecular techniques might have yielded positive results, as shown in other rodent experiments using $\mathrm{PCR}^{4}$. Thus the selective animal models cited by Baker fail to refute the possibility of sexual transmission of Lyme disease in humans.

Baker attempts to use CDC surveillance statistics to further disprove sexual transmission of Lyme disease. He points to the fact that $95 \%$ of reported CDC surveillance cases occur in 12 states in the USA, and that these reported cases are seasonal and have a male and child predominance. As pointed out in our article, however, CDC surveillance cases account for less than $10 \%$ of total Lyme cases (about 30,000 cases out of more than 300,000 annual cases in the USA). Thus the epidemiology of the vast majority of Lyme cases is not covered by CDC surveillance reporting and may differ significantly from the epidemiology reported by the CDC. To support this view, as noted in our study, a recent report from Australia found that the prevalence of Lyme disease cases aligned with the sexually active population on that continent ${ }^{18}$. Baker also questions whether our Lyme disease testing was accurate since it differs from the serological parameters used for CDC surveillance criteria. However the CDC surveillance criteria rely on testing that misses more than half of Lyme disease $\operatorname{cases}^{19}$, and these criteria are not recommended for diagnosis of Lyme disease ${ }^{20}$. The reason why Baker thinks that insensitive and incomplete epidemiology statistics trump microscopy, immunochemistry, molecular hybridization and PCR testing that demonstrates live, culturable Borrelia spirochetes in genital secretions from our Lyme disease patients is unclear. 
Baker bemoans the fact that because of the inconvenient evidence presented in our study, Lyme disease patients may suddenly be concerned about sexual transmission of their disease. Because Baker does not represent or treat patients, he may be unaware that this question is being asked by patients in clinicians' offices every day as spouses and sexual partners become symptomatic. Should Lyme disease patients be concerned about sexual transmission? Absolutely. We currently treat Lyme disease with 60-year-old antibiotics that are marginally effective against a chronic infectious disease ${ }^{1,21}$. Baker and his research associates should focus on the risk of sexual transmission of Borrelia and strive to develop new treatments for this alarming epidemic infection along the lines of the global "Manhattan Project" that has successfully dealt with the HIV/AIDS epidemic $^{2,19}$. Our suffering Lyme disease patients deserve nothing less.

\section{References}

1. Stricker RB, Johnson L: Lyme disease: The next decade. Infect Drug Resist. 2011; 4: 1-9 PubMed Abstract | Free Full Text

2. Stricker RB, Johnson L: Lyme disease: Call for a "Manhattan Project" to combat the epidemic. PLoS Pathog. 2014; 10 (1): e1003796 PubMed Abstract | Free Full Text | Publisher Full Text

3. Gustafson JM, Burgess EC, Wachal MD, Steinberg H: Intrauterine transmission of Borrelia burgdorferi in dogs. Am J Vet Res. 1993; 54 (6): 882-890 PubMed Abstract

4. Burgess EC, Wachal MD, Cleven TD: Borrelia burgdorferi infection in dairy cows, rodents, and birds from four Wisconsin dairy farms. Vet Microbiol. 1993; 35 (1-2): 61-77 PubMed Abstract 5. Schlesinger PA, Duray PH, Burke BA, Steere AC, et al.: Maternal-fetal transmission of the Lyme disease spirochete, Borrelia burgdorferi. Ann Intern Med. 1985; 103 (1): 67-68 PubMed Abstract 6. Carlomagno G, Luksa V, Candussi G, Rizzi GM, et al.: Lyme Borrelia positive serology associated with spontaneous abortion in an endemic Italian area. Acta Eur Fertil. 1988; 19 (5): 279-281 PubMed Abstract

7. Weber K, Bratzke HJ, Neubert U, Wilske B, et al.: Borrelia burgdorferi in a newborn despite oral penicillin for Lyme borreliosis during pregnancy. Pediatr Infect Dis J. 1988; 7 (4): 286-289 PubMed Abstract

8. MacDonald AB: Gestational Lyme borreliosis: implications for the fetus. Rheum Dis Clin North Am. 1989; 15 (4): 657-677 PubMed Abstract

9. Moody KD, Barthold SW: Relative infectivity of Borrelia burgdorferi in Lewis rats by various routes of inoculation. Am J Trop Med Hyg. 1991; 44 (2): 135-139 PubMed Abstract

10. Woodrum JE, Oliver JH: Investigation of venereal, transplacental, and contact transmission of the Lyme disease spirochete, Borrelia burgdorferi, in Syrian hamsters. J Parasitol. 1999; 85 (3): 426430 PubMed Abstract

11. Burgess EC, Amundson TE, Davis JP, Kaslow RA, et al.: Experimental inoculation of Peromyscus spp. with Borrelia burgdorferi: Evidence of contact transmission. Am J Trop Med Hyg. 1986; 35 (2): 355-359 PubMed Abstract

12. Wright SD, Nielsen SW: Experimental infection of the white-footed mouse with Borrelia burgdorferi. Am J Vet Res. 1990; 51 (12): 1980-1987 PubMed Abstract

13. Gustafson JM: The in utero and seminal transmission of Borrelia burgdorferi in Canidae (PhD thesis, University of Wisconsin, Madison; Accessed: December 30, 2014). 1993. Reference Source 14. Lewis rat (Accessed: December 30, 2014). Wikipedia. Reference Source 
15. Golden hamster (Accessed: December 30, 2014). Wikipedia. Reference Source

16. Moody KD, Barthold SW, Terwilliger GA, Beck DS, et al.: Experimental chronic Lyme borreliosis in Lewis rats. Am J Trop Med Hyg. 1990; 42 (2): 165-174 PubMed Abstract

17. Johnson RC, Codner C, Russell M, Duray PH: Experimental infection of the hamster with Borrelia burgdorferi. Ann N Y Acad Sci. 1988; 539: 258-263 Publisher Full Text

18. Mayne PJ: Clinical determinants of Lyme borreliosis, babesiosis, bartonellosis, anaplasmosis and ehrlichiosis in an Australian cohort. Int J Gen Med. 2015; 8: 15-26 Publisher Full Text | Reference

Source

19. Stricker RB, Johnson L: Lyme disease diagnosis and treatment: lessons from the AIDS epidemic. Minerva Med. 2010; 101 (6): 419-425 PubMed Abstract

20. Lyme disease: 2011 case definition (Accessed: December 30, 2014). CDC. Reference Source

21. Borgermans L, Goderis G, Vandevoorde J, Devroey D: Relevance of chronic Lyme disease to family medicine as a complex multidimensional chronic disease construct: a systematic review. Int J Family Med. 2014; 2014. Publisher Full Text | Reference Source

Competing Interests: None

\section{Reader Comment 27 Dec 2014}

Phillip Baker, American Lyme Disease Foundation, USA

The concept of sexual transmission of borreliosis, which has been resurrected recently by Middelveen et al. ${ }^{1}$, was refuted years ago by the well-designed and controlled studies of Moody and Barthold ${ }^{2}$, as well as Woodrum and Oliver ${ }^{3}$, internationally known experts on Lyme disease. These investigators used well-characterized animal models of borreliosis in which infection is much more disseminated and profound than it ever is in humans. It should be noted that, in the United States, Lyme borreliosis has historically been defined as a tick borne infection caused by Borrelia burgdorferi sensu lato ${ }^{4}$.

To determine if borreliosis can be transmitted by direct contact, Moody and Barthold ${ }^{2}$ housed three-day-old - or three-week-old- Lewis rats, deliberately infected with B. burgdorferi, with normal, uninfected rats for 30 days. As expected, all deliberately infected rats continued to be actively infected, 30 days later; however, none of the uninfected rats acquired infection after 30 days of intimate direct contact with their infected house mates.

In other experiments, Moody and Barthold ${ }^{2}$ were unable to demonstrate venereal transmission of borreliosis from seven infected females-or six infected males - to uninfected rats of the opposite sex.

In the work of Woodrum and Oliver ${ }^{3}$, six female Syrian hamsters infected with B.burgdorferi were mated with six uninfected males; conversely, three infected males were mated with six uninfected females. None of the uninfected hamsters became infected after mating with an infected partner of the opposite sex, indicating that borreliosis is not sexually transmitted. These investigators also failed to demonstrate contact transmission of $B$. burgdorferi between infected female -or male- 
hamsters and uninfected hamsters of the opposite sex. Also, it was not possible to transmit borreliosis to uninfected hamsters with urine or feces from infected hamsters.

Sadly, preliminary oral reports of the observations of Middelveen et al. ${ }^{1}$ have already generated an inordinate amount of fear and anxiety within the lay community due to sensationalized reports of their unconfirmed findings by an uncritical - and often naïve - press. This has already caused much harm. To date, I have received numerous inquiries from distraught individuals, wondering if they now should even consider marrying their spouse-to-be for fear of contracting Lyme disease that some mistakenly believe to be incurable. Some fear the possibility of giving birth to an infected or congenitally deformed child, because their spouse or spouse-to-be had been diagnosed and treated for Lyme disease in the past.

To examine the issue of in utero transmission of infection, Moody and Barthold ${ }^{2}$ inoculated pregnant female Lewis rats with viable $B$. burgdorferi, at four days of gestation. All of the inoculated pregnant females became seropositive as expected, and $B$. burgdorferi could be cultured from their spleens at 20 days of gestation; however, their placentas and fetuses were culture negative, indicting the lack of in utero transmission.

Moody and Barthold ${ }^{2}$ used two different experimental protocols to determine if transplacental transmission of $B$. burgdorferi occurs. One protocol involved six non-pregnant infected females that were subsequently mated and became pregnant. Three of the females were allowed to carry to full term, whereas the remaining three were sacrificed just prior to parturition. All offspring and offspring-to-be were found to be culture negative for B. burgdorferi, as well as seronegative for antibody specific for B. burgdorferi, indicating that transplacental transmission of infection does not occur.

In the second protocol, six females were infected via tick bite after becoming pregnant, and were allowed to carry their fetuses to birth; all were negative for infection. The results of these studies likewise failed to provide evidence for the transplacental transmission of naturally acquired borreliosis.

Other investigators examined the possibility of congenital birth defects in humans with Lyme disease by doing a rather large comparative study involving 5,000 infants, half from an area in which Lyme disease was endemic and half as controls from an area without Lyme disease ${ }^{5}$. They found no significant differences in the overall incidence of congenital malformations between the two groups.

In another study, involving 1,500 subjects including controls, no increased risk of giving birth to a child with a congenital heart defect was noted in women who had either been bitten by a tick or had been treated for Lyme disease during or before pregnancy ${ }^{6}$.

Finally, an extensive analysis of the world literature revealed "that an adverse outcome due to maternal infection with $B$. burgdorferi at any point during pregnancy in humans is at most extremely rare" 7 .

In view of the above considerations, the work by Middelveen et al. ${ }^{1}$ is not credible and must be 
view with much skepticism. There is no evidence to indicate that vaginal and seminal secretions provide a suitable environment for the growth of Borrelia ${ }^{8}$ and that the mere presence of Borrelia in vaginal and seminal secretions - if such an observation is indeed valid and can be confirmed independently - results in disease. In the absence of such proof, no conclusions can be drawn as to the sexual transmission of Lyme disease by of B. burgdorferi.

\section{Addendum added in proof:}

It should be noted that the basic epidemiology of vector-borne Lyme disease is strikingly different from that for sexually transmitted diseases (STDs). For example, annual data provided by the CDC indicate that about $95 \%$ of all reported cases of Lyme disease occur in 12 States, and the incidence of Lyme disease is seasonal and peaks with increased tick activity. Also, in the case of STDs, there are more husband-wife paired cases rather than the preponderance of cases of males and children with Lyme disease. Most important, Middelveen et al. state that "... the majority of our study subjects did not meet the positive standard as defined by the CDC surveillance criteria." One must therefore wonder if their study subjects ever had Lyme disease in the first place.

\section{$\underline{\text { References }}$}

1. Middleveen, MJ, Burke, J., Sapi, E., et al. Culture and identification of Borrelia spirochetes in human vaginal and seminal secretions. [v1; ref status: awaiting peer review, http://f1000r.es/4rm] published 19 December 2014, F1000Research 2014, 3:309 (doi: 10.12688/f1000 research.57778.1).

2. Moody, KD, and Barthold, SW. Relative infectivity of Borrelia burgdorferi in Lewis rats by varius routes of inoculation. Amer. J. Trop. Med. Hyg. 1991; 44: 135-139.

3. Woodrum, JE, and Oliver, JH Jr. Investigation of venereal, transplacental, and contact transmission of the Lyme disease sprirochete, Borrelia burgdorferi, in Syrian hamsters. J. Parasitol. 1999: 85, 426-430.

4. Wormser, GP, and O'Connell, S. Treatment of infection caused by Borrelia burgdorferi sensu lato. Expert. Rev. Anti. Infect. Ther. 2011; 9: 245-260.

5. Williams, CL, Strobino, B, Weinstein, A. et al. Maternal Lyme disease; congenital malformations and a cord blood serosurvey in endemic and control areas. Paediatr. Perinat. Epidemiol. 1995; 180: 711-716.

6. Strobino, B, Abid, S, and Gewitz, M. Maternal Lyme disease and congential heart disease: a case control study in an endemic area. Am. J. Obstet. Gynecol. 199; 180: 711-716.

7. Elliot, DJ, Eppes, SC, and Klein, JD. Teratogen Update: Lyme disease. Teratology 2001; 64: 276286.

8. http://www.cdc.gov/lyme/faq/\#sexually 
Competing Interests: No competing interests were disclosed.

The benefits of publishing with F1000Research:

- Your article is published within days, with no editorial bias

- You can publish traditional articles, null/negative results, case reports, data notes and more

- The peer review process is transparent and collaborative

- Your article is indexed in PubMed after passing peer review

- Dedicated customer support at every stage

For pre-submission enquiries, contact research@f1000.com 